ESAIM: PROCEEDINGS AND SURVEYS, September 2019, Vol. 66, p. 61-83

Philippe Helluy, Jean-Marc Hérard and Nicolas Seguin Editors

\title{
ON BAER-NUNZIATO MULTIPHASE FLOW MODELS
}

\author{
M. Hillairet ${ }^{1}$
}

\begin{abstract}
In these notes, we present an analytical tool for the derivation of Baer-Nunziato multiphase flow systems with one velocity. We explain the method in the case of the isentropic Navier Stokes equations. We then apply this method to models with temperature and show the main computations which are necessary to the derivation.
\end{abstract}

\section{INTRODUCTION}

Multiphase flows are involved in many industrial process. In some of them, experimental results are lacking. It is then mandatory to involve theoretical/abstract arguments to derive predictive models. In this note, we describe an analytical tool which aims to help in this task. We focus on multiphase flows for which the mixture holds at a lower scale than observation. This means that each fluid element volume that we consider contains a melange of several phases. In terms of topological distribution of interfaces, this corresponds to "mixed/transitional" or "dispersed" flows in the classification of [9]. In this context, a classical method for deriving multiphase flow models consists in the following steps:

(1) to write local equilibrium equations for all phases;

(2) to fix transfer equations at interfaces;

(3) to perform means and obtain average equations.

Several classical difficulties arise [6,9]. First, the nature of the mean operators involved in the last step is a current subject of debate. Second, the obtained averaged equations remain to be closed. This leads to the introduction of adequate closure equations, that are also subject to debate. Herein, we consider simplified cases in which we can interpret the mixture model obtained after steps (1) and (2) of the method as the equations for a single-phase fluid whose macroscopic fields (density, velocity, pressure, internal energy) exhibit discontinuities (corresponding to jumps of the values of these quantities in the different components of the mixture). Then, the third step (3) of the derivation can be seen as a homogenization problem for oscillating solutions to the single-phase model.

From the analytical stand-point, the analysis of oscillations in single-phase models is a classical issue related to the construction of weak solutions. We describe now the approach of $[7,12]$ in the case of viscous compressible models for the simplified $1 \mathrm{D}$ case. Let consider the problem of finding $(\rho, u)$ solution to

$$
\begin{aligned}
\partial_{t} \rho+\partial_{x}(\rho u) & =0, \\
\partial_{t}(\rho u)+\partial_{x}\left(\rho u^{2}\right) & =\partial_{x}\left(\mu \partial_{x}-p\right), \\
p & =a \rho^{\gamma},
\end{aligned}
$$

\footnotetext{
${ }^{1}$ Institut Montpelliérain Alexander Grothendieck, Université de Montpellier, Place Eugène Bataillon, 34095 Montpellier Cedex

5 France (e-mail: matthieu.hillairet@umontpellier.fr)
}

(C) EDP Sciences, SMAI 2019

This is an Open Access article distributed under the terms of the Creative Commons Attribution License (http://creativecommons.org/licenses/by/4.0), which permits unrestricted use, distribution, and reproduction in any medium, provided the original work is properly cited. 
where $\mu, a>0$ are constant parameters and $\gamma>1$ is a given exponent. For simplicity, let consider periodic solutions to this system so that the above equations are satisfied on the torus $\mathbb{T}=\mathbb{R} / 2 \pi \mathbb{Z}$. Standard formal multiplier arguments show that a reasonable solution to (1)-(2)-(3) has to converve the fluid mass:

$$
\int_{\mathbb{T}} \rho(t, x) \mathrm{d} x=\int_{\mathbb{T}} \rho(0, x) \mathrm{d} x
$$

and dissipate the total energy:

$$
\mathcal{E}(t)=\int_{\mathbb{T}}\left[\rho(t, x) \frac{|u(t, x)|^{2}}{2}+\frac{a}{\gamma-1} \rho^{\gamma}(t, x)\right] \mathrm{d} x,
$$

with an a priori dissipation rate:

$$
\frac{\mathrm{d}}{\mathrm{d} t} \mathcal{E}(t)=-\int_{\mathbb{T}} \mu\left|\partial_{x} u(t, x)\right|^{2} \mathrm{~d} x
$$

In the multidimensional setting, constructing global classical solutions to (1)-(2)-(3) seems out of reach. Then, one needs to turn to the construction of weak solutions, in the sense that these solutions satisfy

- (1)-(2)-(3) in the sense of distributions,

- conservation law (4) and energy estimate (6).

A classical method for constructing such solutions is the compactness method: one first constructs global solutions to a regularized system $\left(\rho_{n}, u_{n}\right)_{n \in \mathbb{N}}$ and then, letting the regularization parameter $n \rightarrow \infty$, shows that it is possible to extract a cluster point which is a weak solution to (1)-(2)-(3). In order to answer this latter question, the main issue concerns possible oscillations of the density (see also [13]): one must show that the limit pressure is related to the limit density via the state equation (3). To prove this property, one possibility is to introduce Young measures to describe the propagation of oscillations in the solutions and derive an entropy estimate showing that if there are no oscillations initially - i.e. that the Young measures are concentrated on a graph - then the same holds for all times. A crucial intermediate step consists in finding combinations of unknowns which enjoy better compactness properties (see the properties of the effective viscous flux $[8,13]$ ). To derive multiphase systems, one extends these computations to a larger class of Young measures.

In this note, we first construct the class of Young measures which may represent multiphase flows. Then, we detail the results that are obtained in [1]. In particular, we recall the main key-question/ingredients that underly the derivation of a multiphase system. In the last section, we consider a temperature-dependent model and we perform the formal computations that enable to guess the multiphase flow system that this method would enable to derive.

\section{Modeling a multiphase flow via Young measures}

In this section, we illustrate the relations between ocillations and multiphase flows on a particular case. We then depict briefly how Young measures enable to describe oscillations and thus represent multiphase flows.

\subsection{Relations between oscillations and multiphase flows}

Let consider the mixture of two phases, say a liquid and a gas. Assume for simplicity that the mixture fills the container $\Omega=[0,1]^{d}$ in $\mathbb{R}^{d}$. There is no restriction on the dimension $d$ at this level. We denote $\rho_{l}: \Omega_{l} \rightarrow[0, \infty)$ (resp. $\rho_{g}: \Omega_{g} \rightarrow[0, \infty)$ ) the density field of the liquid (resp. of the gas). This density-field is defined on the domain $\Omega_{l}$ occupied by the liquid (resp. $\Omega_{g}$ occupied by the gas). Assuming that the phases are immiscible and that there is no vacuum in the domain, we have:

$$
\bar{\Omega}_{g} \cup \bar{\Omega}_{l}=\bar{\Omega}, \quad \Omega_{g} \cap \Omega_{l}=\emptyset .
$$




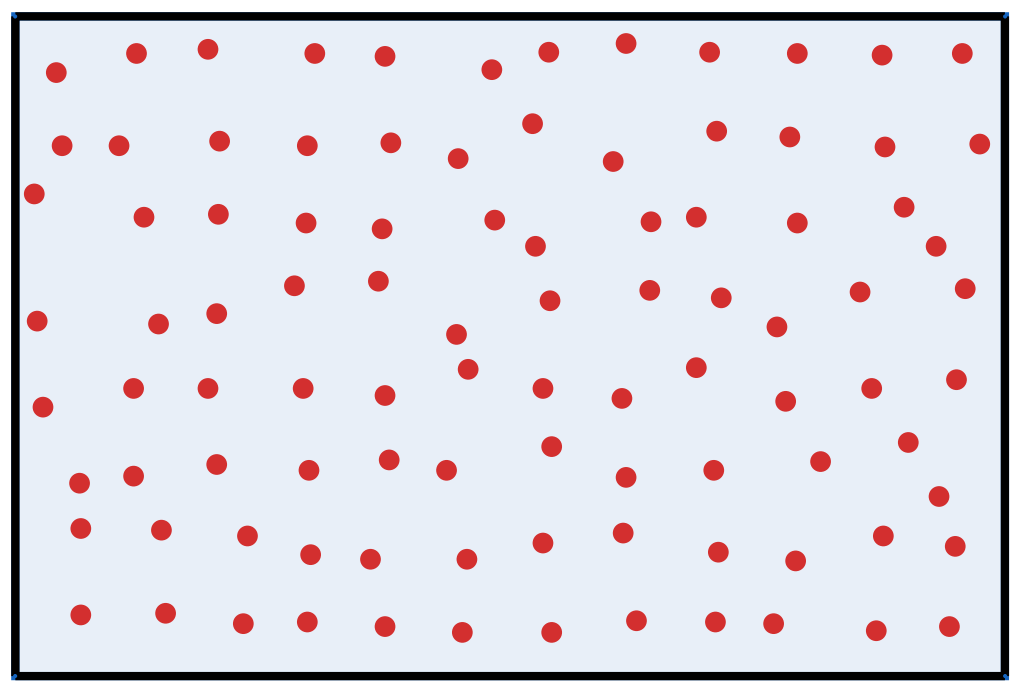

Figure 1. Example of bubbly flow : liquid in blue and gas bubbles in red.

We may then introduce the composite density:

$$
\rho(x)=\rho_{l}(x) \mathbf{1}_{\Omega_{l}}(x)+\rho_{g}(x) \mathbf{1}_{\Omega_{g}}(x)= \begin{cases}\rho_{l}(x), & \text { if } x \in \Omega_{l} \\ \rho_{g}(x), & \text { if } x \in \Omega_{g}\end{cases}
$$

We focus on models such that any infinitesimal volume contains both phases. For instance, an application we have in mind is a bubbly flow where $\Omega_{g}$ is the union of a large number of small gas drops inside the liquid. To describe this particular case, let introduce a parameter $\varepsilon>0$ (small) and denote $\left(T_{k}^{\varepsilon}\right)_{k \in \mathcal{K}^{\varepsilon}}$ a covering of $[0,1]^{d}$ with cubes of width $\varepsilon$. Each cell $T_{k}^{\varepsilon}$ contains a drop $D_{k}^{\varepsilon} \Subset T_{k}^{\varepsilon}$ of volume $\alpha_{g} \varepsilon^{d}$, say $D_{k}^{\varepsilon}$ is the cube with center $x_{k}^{\varepsilon}$ and radius $\alpha_{g}^{1 / d} \varepsilon$, where $x_{k}^{\varepsilon} \in T_{k}^{\varepsilon}$ and $\alpha_{g} \in(0,1)$ represents the volume fraction of the liquid phase. If we assume furthermore that $\rho_{g}$ and $\rho_{l}$ are constant, we obtain:

$$
\mathbf{1}_{\Omega_{g}}=\sum_{k \in \mathcal{K}^{\varepsilon}} \mathbf{1}_{D_{k}^{\varepsilon}} \mathbf{1}_{\Omega_{l}}=\sum_{k \in \mathcal{K}^{\varepsilon}} \mathbf{1}_{T_{k}^{\varepsilon} \backslash \bar{D}_{k}^{\varepsilon}},
$$

and

$$
\rho=\rho^{\varepsilon}=\rho_{g} \sum_{k \in \mathcal{K}^{\varepsilon}} \mathbf{1}_{D_{k}^{\varepsilon}}+\rho_{l} \sum_{k \in \mathcal{K}^{\varepsilon}} \mathbf{1}_{T_{k}^{\varepsilon} \backslash \bar{D}_{k}^{\varepsilon}} .
$$

Since we assume $\varepsilon<<1$, we want to compute a description of the mixture by finding a limit to $\left(\rho^{\varepsilon}\right)_{\varepsilon>0}$ when $\varepsilon \rightarrow 0$. The basics of the multiphase flow modeling is contained in the following proposition:

Proposition 1. In the example described above, let denote $\bar{\rho}:=\alpha_{g} \rho_{g}+\left(1-\alpha_{g}\right) \rho_{l}$. There holds:

- $\rho^{\varepsilon} \rightarrow \bar{\rho}$ in $L^{p}(\Omega)-w$ for arbitrary $p \in[1, \infty)$,

- $\rho^{\varepsilon} \rightarrow \bar{\rho}$ in $L^{p}(\Omega)$ for $p \in[1, \infty]$ if and only if $\rho_{g}=\rho_{l}$.

Proof. First, we note that since $\Omega$ is bounded and $\rho_{g}, \rho_{l}$ are constant, the densities $\left(\rho^{\varepsilon}\right)_{\varepsilon>0}$ are bounded in $L^{p}(\Omega)$ for arbitrary $p \in[1, \infty]$. 


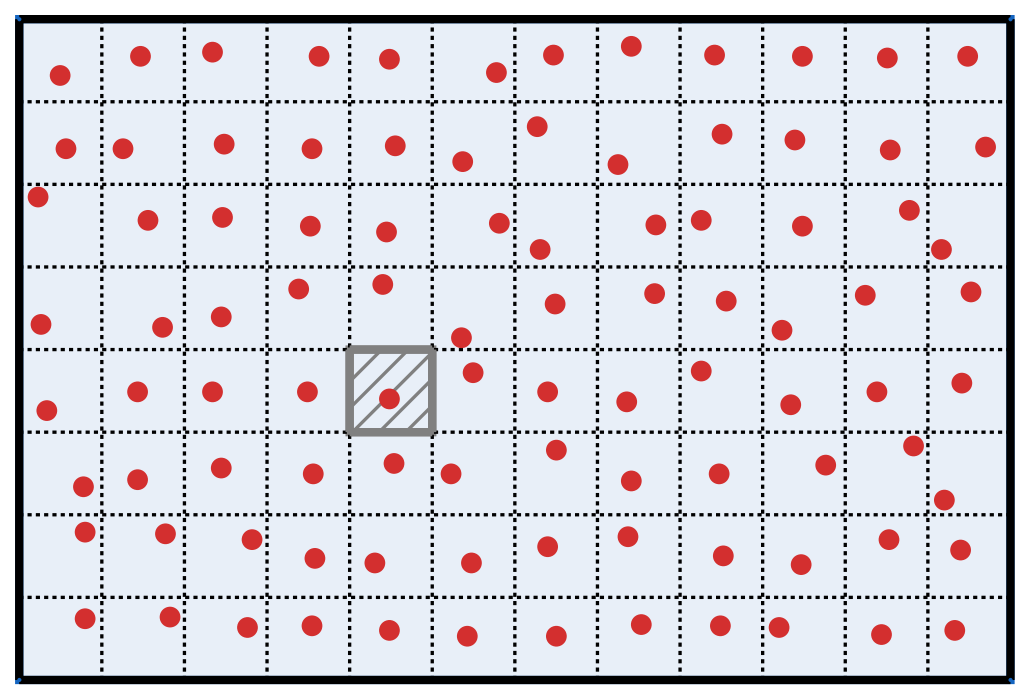

Figure 2. Cell decomposition of an almost periodic bubbly flow.

Given $\varphi \in C_{c}^{\infty}(\Omega)$ we have:

$$
\begin{aligned}
\int_{\Omega} \rho^{\varepsilon} \varphi & =\sum_{k \in \mathcal{K}^{\varepsilon}} \rho_{g} \int_{D_{k}^{\varepsilon}} \varphi+\rho_{l} \int_{T_{k}^{\varepsilon} \backslash \bar{D}_{k}^{\varepsilon}} \varphi \\
& =\sum_{k \in \mathcal{K}^{\varepsilon}}\left(\alpha_{g} \rho_{g}+\left(1-\alpha_{g}\right) \rho_{l}\right) \int_{T_{k}^{\varepsilon}} \varphi+O(\varepsilon),
\end{aligned}
$$

which entails the weak convergence.

On the other hand, we obtain the necessary condition for the strong convergence in $L^{1}$ to hold, which entails the other result. We have:

$$
\begin{aligned}
\int_{\Omega}\left|\rho^{\varepsilon}-\left(\alpha_{g} \rho_{g}+\left(1-\alpha_{g}\right) \rho_{l}\right)\right| & =\sum_{k \in \mathcal{K}^{\varepsilon}}\left|D_{k}^{\varepsilon}\right|\left(1-\alpha_{g}\right)\left|\rho_{g}-\rho_{l}\right|+\left|T_{k}^{\varepsilon} \backslash \overline{D_{k}^{\varepsilon}}\right| \alpha_{g}\left|\rho_{g}-\rho_{l}\right| \\
& \sim \alpha_{g}\left(1-\alpha_{g}\right)\left|\rho_{g}-\rho_{l}\right| .
\end{aligned}
$$

Since $\left.\alpha_{g} \in\right] 0,1\left[\right.$, this term converges to 0 if and only if $\rho_{g}=\rho_{l}$.

If $\rho_{g} \neq \rho_{l}$, the lack of strong convergence is due to the strong oscillations of $\rho^{\varepsilon}$. These oscillations are related to the multiphase nature of the flow under consideration. Introducing the Young measures - which enable to describe the density oscillations - we expect to be able to represent the different phases in the flow. Before a brief description of these Young measures, several remarks are in order. First, we emphasize that we need a tool enabling to split the informations on the partial densities $\rho_{g}$ and $\rho_{l}$ and volume fraction $\alpha_{g}, \alpha_{l}=1-\alpha_{g}$. Indeed, though these quantities are known in the above construction, in a realistic derivation, we may only have access to mean quantities such as $\bar{\rho}$. Second, we note that we restrict here to density-oscillations and shall obtain one-velocity models only. This is related to the viscous setting under consideration below. Similar tools can be constructed to describe velocity oscillations in the inviscid case. This leads to further theoretical difficulties. We refer the reader to [4] for more details. 


\subsection{Reminder on Young measures}

The theory of Young measures or generalized functions is introduced by [3,14] for the analysis of partial differential equations. The key-idea is to identify a function with a positive radon measure. One possible motivation is the bubbly-flow example above in which the lack of strong-compactness of the density sequence $\left(\rho^{\varepsilon}\right)_{\varepsilon>0}$ is due to the oscillations of the indicator functions $\mathbf{1}_{\Omega_{l}}$ and $\mathbf{1}_{\Omega_{g}}$ whose convergences are better computed in the space of measures.

To describe density oscillations, one proposes that a density-field $\rho \in L^{1}(\Omega)$ is identified with a measure $\nu:=\nu_{\rho}$ on $\Omega \times \mathbb{R}$ as defined by:

$$
\left\langle\nu_{\rho}, B\right\rangle=\int_{\Omega} B(x, \rho(x)) \mathrm{d} x, \quad \forall B \in C_{b}(\Omega \times \mathbb{R}) .
$$

We note that this identification yields that $\nu_{\rho}$ is positive and that its mass is $|\Omega|$. In particular, in the case $\Omega=[0,1]^{d}$, we obtain a probability measures. The above identification can be interpreted as seeing the mapping $x \mapsto(x, \rho(x))$ as a random variable on $\Omega$ and constructing the associated law $\nu_{\rho}$. One good feature of this identification is that we may apply directly results on random variables to yield:

Lemma 2. Let $\left(\rho^{\varepsilon}\right)_{\varepsilon>0}$ be a bounded sequence in $L^{1}(\Omega)$. Let denote $\left(\nu^{\varepsilon}\right)_{\varepsilon>0}$ the associated sequence of measures. Then, up to the extraction of a subsequence, we have that $\left(\nu^{\varepsilon}\right)_{\varepsilon>0}$ converges to a positive measure $\bar{\nu}$ in the sense that:

$$
\lim _{\varepsilon \rightarrow 0}\left\langle\nu^{\varepsilon}, B\right\rangle=\langle\bar{\nu}, B\rangle, \quad \forall B \in C_{b}(\Omega \times \mathbb{R}) .
$$

This result is a consequence to the fact that, with the assumptions of this lemma, the random variables $x \mapsto\left(x, \rho^{\varepsilon}(x)\right)$ are uniformly tight. We recall that, with this abstract result at-hand, we can then apply the disintregation theorem to construct a measurable mapping $x \mapsto \bar{\nu}_{x}$ where $\bar{\nu}_{x}$ is a probability measure on $\mathbb{R}$ so that:

$$
\langle\bar{\nu}, B\rangle=\int_{\Omega}\left\langle\bar{\nu}_{x}, B(x, \cdot)\right\rangle \mathrm{d} x .
$$

The new mapping $x \mapsto \bar{\nu}_{x}$ will be called generalized-functions while we shall keep the name Young measures for $\bar{\nu}$. To give a more "computable" approach to $\bar{\nu}_{x}$ we also provide the following characterization:

Lemma 3. Under the assumptions of Lemma 2, the generalized functions $x \mapsto \bar{\nu}_{x}$ satisfy:

$$
\lim _{\varepsilon \rightarrow 0} \beta\left(\rho_{\varepsilon}\right)=\left(x \mapsto\left\langle\bar{\nu}_{x}, \beta\right\rangle\right) \quad \text { a.e. }
$$

for arbitrary $\beta \in C_{b}(\mathbb{R})$.

Proof. It suffices to remark that for arbitrary $\beta \in C_{b}(\mathbb{R})$, the sequence $\left(\beta\left(\rho^{\varepsilon}\right)\right)_{\varepsilon>0}$ is bounded in $L^{\infty}(\Omega)$ and converges weakly (up to the extraction of a subsequence) to some $\bar{\beta} \in L^{\infty}(\Omega)$. For arbitrary $\phi \in C_{c}^{\infty}(\Omega)$, we have then:

$$
\lim _{\varepsilon \rightarrow 0} \int_{\Omega} \beta\left(\rho^{\varepsilon}(x)\right) \phi(x) \mathrm{d} x=\int_{\Omega} \bar{\beta}(x) \phi(x) \mathrm{d} x
$$

and, on the other hand, considering $B(x, \xi)=\phi(x) \beta(\xi) \in C_{b}(\Omega \times \mathbb{R})$ and applying the definition of $x \mapsto \bar{\nu}_{x}$ :

$$
\begin{aligned}
\lim _{\varepsilon \rightarrow 0} \int_{\Omega} \beta\left(\rho^{\varepsilon}(x)\right) \phi(x) \mathrm{d} x & =\lim _{\varepsilon \rightarrow 0} \int_{\Omega} B\left(x, \rho_{\varepsilon}(x)\right) \mathrm{d} x \\
& =\langle\bar{\nu}, B\rangle=\int_{\Omega}\left\langle\bar{\nu}_{x}, \beta\right\rangle \phi(x) \mathrm{d} x .
\end{aligned}
$$


The second construction suits better the analysis of partial differential equations. Indeed, with this construction, the computation of the Young measures reduces to compute limits of functions of the sequence of densities. Computing then equations for Young measures can be related to the theory of renormalized solutions (see [5]). We keep in what follows the notations introduced in this last proof for variables in the space $\Omega \times \mathbb{R}($ on which will be defined our Young measures). We denote by $x \in \Omega$ the space variable and by $\xi \in \mathbb{R}$ the "density" variable.

As a first application, we show that the Young measures enable to characterize completely the limit in the bubbly-flow example of Section 1.1. Indeed, we have the following proposition:

Proposition 4. Keeping the notations of Section 1.1, the measures associated with the densities $\left(\rho^{\varepsilon}\right)_{\varepsilon>0}$ converge toward the generalized function:

$$
x \mapsto \bar{\nu}_{x}=\alpha_{g} \delta_{\rho_{g}}+\left(1-\alpha_{g}\right) \delta_{\rho_{g}}
$$

We denote in this proposition $\delta_{\xi_{0}}$ the Dirac measure centered in $\xi_{0}$. This result writes also : for arbitrary $B \in C_{b}(\Omega \times \mathbb{R})$

$$
\langle\bar{\nu}, B\rangle=\int_{\Omega}\left(\alpha_{g} B\left(x, \rho_{g}\right)+\left(1-\alpha_{g}\right) B\left(x, \rho_{l}\right)\right) \mathrm{d} x .
$$

Proof. We apply the method proposed by Lemma 3 to compute the limit. Given $\beta \in C_{b}(\mathbb{R})$ and $\varphi \in C_{c}^{\infty}(\Omega)$ we have:

$$
\int_{\Omega} \beta\left(\rho^{\varepsilon}\right) \varphi(x) \mathrm{d} x=\beta\left(\rho_{g}\right) \int_{\Omega} \varphi(x) \mathbf{1}_{\Omega_{g}}+\beta\left(\rho_{l}\right) \int_{\Omega} \varphi(x) \mathbf{1}_{\Omega_{l}} .
$$

Here standard arguments show that:

$$
\begin{aligned}
\int_{\Omega} \varphi(x) \mathbf{1}_{\Omega_{g}} & =\sum_{k \in \mathcal{K}^{\varepsilon}} \alpha_{g} \varepsilon^{3} \varphi\left(x_{k}^{\varepsilon}\right)+O(\varepsilon) \\
& =\alpha_{g} \int_{\Omega} \varphi(x) \mathrm{d} x+O(\varepsilon)
\end{aligned}
$$

Similarly, we obtain:

so that, in the limit $\varepsilon \rightarrow 0$, we obtain:

$$
\int_{\Omega} \varphi(x) \mathbf{1}_{\Omega_{l}}=\left(1-\alpha_{g}\right) \int_{\Omega} \varphi(x) \mathrm{d} x+O(\varepsilon),
$$

$$
\lim _{\varepsilon \rightarrow 0} \int_{\Omega} \beta\left(\rho^{\varepsilon}\right) \varphi(x) \mathrm{d} x=\left[\alpha_{g} \beta\left(\rho_{g}\right)+\left(1-\alpha_{g}\right) \beta\left(\rho_{l}\right)\right] \int_{\Omega} \varphi(x) \mathrm{d} x .
$$

Clearly, Proposition 4 extends to more general cases. We can perform similar computations if $\rho_{g}, \rho_{l}$ and $\alpha_{g}$ are not constant but restrictions of continuous functions defined on $\bar{\Omega}$. But, we may also obtain comparable results in case the cells $\left(T_{k}^{\varepsilon}\right)$ contain more than two species. With the observations of this section, we obtain that the class of generalized functions representing a multiphase flow containing $k$ phases is the set

$$
\mathcal{C}_{k}:=\left\{x \mapsto \sum_{i=1}^{k} \alpha_{i}(x) \delta_{\rho_{i}(x)} \quad \text { s.t. } \quad \alpha_{i} \geqslant 0 \text { and } \sum_{i=1}^{k} \alpha_{i}=1\right\}
$$

We have also the following method to derive multiphase flows with $k \geqslant 2$ phases:

(1) to write local equilibrium equations for all phases;

(2) to fix transfer equations at interfaces. 
After these two steps, we interpret the obtained system as a single-phase system for the composite unknowns (see for example the definition of $\bar{\rho}$ ). It remains then

(3) to compute a homogenized single-phase system by introducing Young measures or generalized functions;

(4) to compute solutions to the obtained system prescribing furthermore that the generalized functions are initially in $\mathcal{C}_{k}$.

\section{Isentropic Compressible Navier Stokes System}

To show one application, we report on [2], where we consider the mixture of different phases modeled by the isentropic Navier Stokes system.

\subsection{Microscopic model.}

We start here with the two first steps in case of two viscous compressible phases. From now on, when there are only two phases, we choose to identify the phases with the two indices + and - . We keep numbers for more complicated cases and indices $g, l$ for the particular case of a liquid-gas mixture. In this modeling step, we consider a 3D configuration.

(1) Local equilibrium equations for phases. Assuming that the flows are isentropic, we describe the time evolution of the phases by introducing their density/velocity-field $\left(\rho_{+}, u_{+}\right)$and $\left(\rho_{-}, u_{-}\right)$respectively. We also assume that viscosity is not to be neglected in both phases and we introduce $\left(\mu_{ \pm}, \lambda_{ \pm}\right)$and $p_{ \pm}$the respective viscosities and pressure laws of the phases. For $k=+,-$ we can then model the behavior of phase $k$ by saying that the triplet $\left(\rho_{k}, u_{k}\right)$ is a solution to the compressible Navier Stokes equations

$$
\begin{aligned}
\partial_{t} \rho_{k}+\operatorname{div}\left(\rho_{k} u_{k}\right) & =0, \\
\partial_{t}\left(\rho_{k} u_{k}\right)+\operatorname{div}\left(\rho_{k} u_{k} \otimes u_{k}\right) & =\operatorname{div} \Sigma_{k},
\end{aligned}
$$

on the domain $\Omega_{k}(t)$, filled by the phase $k$, and with the constitutive equations:

$$
\begin{aligned}
\Sigma_{k} & =\mu_{k}\left(\nabla u_{k}+\nabla^{\top} u_{k}\right)+\left(\lambda_{k} \operatorname{div} u_{k}-p_{k}\right) \mathbb{I}_{3}, \\
p_{k} & =p_{k}\left(\rho_{k}\right), \quad \mu_{k}=\mu_{k}\left(\rho_{k}\right), \quad \lambda_{k}=\lambda_{k}\left(\rho_{k}\right) .
\end{aligned}
$$

We emphasize that the constitutive equations may differ in both phases. In what follows, we denote the composite unknowns

$$
\rho=\rho_{+} \mathbf{1}_{\Omega_{+}}+\rho_{-} \mathbf{1}_{\Omega_{-}}, \quad u=u_{+} \mathbf{1}_{\Omega_{+}}+u_{-} \mathbf{1}_{\Omega_{-}} .
$$

(2) Interface equations. By convention, there holds:

$$
\Omega_{+} \cap \Omega_{-}=\emptyset \quad \overline{\Omega_{+}} \cup \overline{\Omega_{-}}=\bar{\Omega} .
$$

Though we do not write it explicitly, the domain of each phase depends on time. We prescribe now the timeevolution of their interfaces. We assume the two phases do not slip on each other. On $\partial \bar{\Omega}_{+} \cap \partial \bar{\Omega}_{-}$we have then $u_{+}=u_{-}$and the interface moves with the velocity $u_{I}=u_{+}=u_{-}$.

We fix now the phase interactions at their interfaces. First, we assume that there is no mass transfer. Standard computations on continuity equations entail that the composite density satisfies:

$$
\partial_{t} \rho+\operatorname{div}(\rho u)=0 \quad \text { on } \Omega .
$$

Second, we assume continuity of normal stress. Again, classical assumptions entail then that:

$$
\partial_{t}(\rho u)+\operatorname{div}(\rho u \otimes u)=\operatorname{div} \Sigma .
$$


where

$$
\Sigma=\Sigma_{+} \mathbf{1}_{\Omega_{+}}+\Sigma_{-} \mathbf{1}_{\Omega_{-}} .
$$

At this point, we remark that the values of $\Sigma_{+}$and $\Sigma_{-}$differ only because of the values of the viscosity coefficients $\mu, \lambda$ and pressure law $p$.

We assume then that the two phases are characterized by the fact that their densities range two nonoverlapping intervals $I_{+}$and $I_{-}$. We can then encode the variation of viscosities by seeing the viscosities as density-dependent. Namely, we construct functions $\tilde{\mu}, \tilde{\lambda}$ such that:

$$
\tilde{\mu}(r)=\left\{\begin{array}{ll}
\mu_{+}, & \text {if } r \in I_{+}, \\
\mu_{-}, & \text {if } r \in I_{-},
\end{array} \quad \tilde{\lambda}(r)= \begin{cases}\lambda_{+}, & \text {if } r \in I_{+}, \\
\lambda_{-}, & \text {if } r \in I_{-},\end{cases}\right.
$$

and we perform a similar construction for pressure law:

$$
\tilde{p}(r)= \begin{cases}p_{+}(r), & \text { if } r \in I_{+} \\ p_{-}(r), & \text { if } r \in I_{-}\end{cases}
$$

We have thus the global constitutive equations:

$$
\begin{aligned}
\Sigma & =\mu\left(\nabla u+\nabla^{\top} u\right)+(\lambda \operatorname{div} u-p) \mathbb{I}_{3}, \\
p & =\tilde{p}(\rho), \quad \mu=\tilde{\mu}(\rho), \quad \lambda=\tilde{\lambda}(\rho) .
\end{aligned}
$$

The simplified multiphase flow under consideration here is then modelled by the single-phase system (7)-(8)(9)-(10) satisfied by the composite unknowns $(\rho, u)$.

For the next steps of the analysis, we turn to the one-dimensional analogue system:

$$
\begin{aligned}
\partial_{t} \rho+\partial_{x}(\rho u) & =0 \\
\partial_{t}(\rho u)+\partial_{x}\left(\rho u^{2}\right) & =\partial_{x}\left(\mu \partial_{x} u\right)-\partial_{x} p \\
p & =\tilde{p}(\rho), \quad \mu=\tilde{\mu}(\rho),
\end{aligned}
$$

in the periodic setting.

\subsection{Analysis of the homogenization problem - main result}

We proceed with the two last steps corresponding to the following homogenization problem. We consider a family $\left(\rho^{n}, u^{n}, p^{n}\right)_{n \in \mathbb{N}}$ of solutions to (11)-(12)-(13) on $(0, T) \times \mathbb{T}$ for some $T>0$. Introducing the Young measures $\bar{\nu}$ associated with the sequence of densities $\left(\rho^{n}\right)_{n \in \mathbb{N}}$, we aim at computing a limit $(\bar{\nu}, \bar{u}, \bar{p})$ to the sequence $\left(\nu^{n}, u^{n}, p^{n}\right)_{n \in \mathbb{N}}$ when $n \rightarrow \infty$. Most of all, we aim to compute a system satisfied by this limit. It remains then to consider an associated generalized function $x \mapsto \bar{\nu}_{x}$ such that its initial value $x \mapsto \bar{\nu}_{x}^{0}$ represents a multiphase flow, i.e. $x \mapsto \bar{\nu}_{x}^{0} \in \mathcal{C}_{k}$ for some $k \geqslant 2$.

In [2], we obtain a multiphase system, corresponding in the case $k=2$ to the following bi-fluid system:

$$
\begin{aligned}
& \partial_{t} \alpha_{+}+\bar{u} \partial_{x} \alpha_{+}=\frac{\alpha_{+} \alpha_{-}}{\alpha_{-} \mu_{+}+\alpha_{+} \mu_{-}}\left[\left(p_{+}-p_{-}\right)+\left(\mu_{-}-\mu_{+}\right) \partial_{x} \bar{u}\right] \\
& \partial_{t}\left(\alpha_{+} \rho_{+}\right)+\partial_{x}\left(\alpha_{+} \rho_{+} \bar{u}\right)=0 \\
& \partial_{t} \bar{\rho}+\partial_{x}(\bar{\rho} \bar{u})=0 \\
& \partial_{t}(\bar{\rho} \bar{u})+\partial_{x}\left(\bar{\rho} \bar{u}^{2}\right)=\partial_{x}\left(m \partial_{x} \bar{u}\right)-\partial_{x} \bar{p}
\end{aligned}
$$


with the compatibility condition

$$
\alpha_{+}+\alpha_{-}=1
$$

and the constitutive equation:

$$
\begin{aligned}
& \mu_{+}=\mu_{+}\left(\rho_{+}\right), \quad \mu_{-}=\mu_{-}\left(\rho_{-}\right), \quad p_{+}=p_{+}\left(\rho_{+}\right), \quad p_{-}=p_{-}\left(\rho_{-}\right) \\
& m=\frac{\mu_{+} \mu_{-}}{\alpha_{+} \mu_{-}+\alpha_{-} \mu_{+}}, \quad \bar{\rho}=\alpha_{+} \rho_{+}+\alpha_{-} \rho_{-}, \quad \bar{p}=\frac{\alpha_{+} p_{+} \mu_{-}+\alpha_{-} p_{-} \mu_{+}}{\alpha_{+} \mu_{-}+\alpha_{-} \mu_{+}} .
\end{aligned}
$$

We recall that $\left(p_{+}, \mu_{+}\right)$and $\left(p_{-}, \mu_{-}\right)$are the respective state equations for both phases. The unknowns in this system are $\left(\left(\alpha_{+}, \rho_{+}\right),\left(\alpha_{-}, \rho_{-}\right), u\right)$. A more precise statement of this justification is the following theorem:

Theorem 5. Assume that the above construction yields viscosity/pressure laws such that:

$$
\begin{array}{ll}
\tilde{p}^{\prime} \geqslant 0, & \tilde{p}(s)=a s^{\gamma} \text { for large } s, \\
\tilde{\mu}(s) \geqslant \mu^{0}, & \forall s \in[0, \infty) .
\end{array}
$$

Let $\left(\rho_{0}^{n}, u_{0}^{n}\right)_{n \in \mathbb{N}}$ be a sequence of periodic initial data such that

- $\rho_{0}^{n} \in L^{\infty}(\mathbb{T})$ with

$$
\frac{1}{C_{0}} \leqslant \inf _{\mathbb{T}} \rho_{0}^{n}(x) \leqslant \sup _{\mathbb{T}} \rho_{0}^{n}(x) \leqslant C_{0}, \quad \forall n \in \mathbb{N},
$$

- $u_{0}^{n} \in H^{1}(\mathbb{T})$ with

$$
\left\|u_{0}^{n}\right\|_{H^{1}(\mathbb{T})} \leqslant C_{0}, \quad \forall n \in \mathbb{N},
$$

for a constant $C_{0}$. Assume furthermore that there exists $\left(\left(\alpha_{+}^{0}, \rho_{+}^{0}\right),\left(\alpha_{-}^{0}, \rho_{-}^{0}\right)\right) \in\left(L^{\infty}(\mathbb{T})\right)^{4}$ and $u^{0} \in H^{1}(\mathbb{T})$ such that

- the generalized function $x \mapsto \bar{\nu}_{x}^{0}$ associated with the sequence of initial densities $\left(\rho_{0}^{n}\right)_{n \in \mathbb{N}}$ satisfies:

$$
\bar{\nu}_{x}=\alpha_{+}^{0}(x) \delta_{\rho_{+}^{0}(x)}+\alpha_{-}^{0}(x) \delta_{\rho_{-}^{0}(x)} \quad \text { a.e. }
$$

- $u_{0}^{n}$ converges weakly to $u^{0}$ in $H^{1}(\mathbb{T})$

Then there exists $T>0$ and a sequence of solutions $\left(\rho^{n}, u^{n}\right)_{n \in \mathbb{N}}$ to (11)-(12)-(13) on $(0, T)$ with initial data $\left(\rho_{0}^{n}, u_{0}^{n}\right)_{n \in \mathbb{N}}$ that converges to a solution $\left(\left(\alpha_{+}, \rho_{+}\right),\left(\alpha_{-}, \rho_{-}\right), u\right)$ to $(14)-(15)-(17)-(18)-(19)-(20)$ with initial data $\left(\left(\alpha_{+}^{0}, \rho_{+}^{0}\right),\left(\alpha_{-}^{0}, \rho_{-}^{0}\right), u^{0}\right)$.

We emphasize that, in the assumptions of this theorem, we consider the already constructed extrapolated pressure law $\tilde{p}$ and viscosity law $\tilde{\mu}$. This may seem to have an impact on the result. However, if $p_{+}$and $p_{-}$or $\mu_{+}$and $\mu_{-}$satisfy both the corresponding assumptions, we can always construct extrapolated laws that match these assumptions. Futhermore, the computed multiphase system depends only on the value of the extrapolated laws on the intervals to which $\rho_{+}$and $\rho_{-}$belong.

\subsection{Analysis of the homogenization problem - key issues}

We do not give herein a full proof of Theorem 5 . We raise only the critical issues and let the reader refer to [2] for more details. For this, let take for granted the construction of the solutions $\left(\rho^{n}, u^{n}\right)_{n \in \mathbb{N}}$ to (11)-(12)-(13). With the boundedness assumption on the initial data, one may reasonably consider that the associated sequence of solutions is bounded in the energy-sense:

$$
\sup _{n \in \mathbb{N}} \sup _{t \in(0, T)} \mathcal{E}\left[\rho^{n}, u^{n}\right](t)+\int_{0}^{t} \int \mu^{n}\left|\partial_{x} u^{n}\right|^{2} \leqslant E_{0},
$$


for some strictly positive constant $E_{0}$. We recall that, in this case where $p$ is no longer a power of the density, the energy reads:

$$
\mathcal{E}[\rho, u](t)=\int_{\mathbb{T}}\left[\frac{1}{2} \rho(t, x)|u(t, x)|^{2}+q(\rho(t, x))\right] \mathrm{d} x,
$$

where $q$ is associated with the extended pressure law via the relation:

$$
\frac{\mathrm{d}}{\mathrm{d} z}\left[\frac{q(z)}{z}\right]=\frac{p(z)}{z^{2}}
$$

The important property is that, under the assumption that $p^{\prime} \geqslant 0$ this term can be made positive and the energy $\mathcal{E}$ remains positive definite. We also point out that the viscosity is no longer constant and depends on $n$. We denote $\mu^{n}:=\mu\left(\rho^{n}\right)$. We also add an a priori bound for simplicity. In the problem under consideration, vacuum/concentration of the density is not a first issue, so we enforce the following bounds on the densities:

$$
\sup _{n \in \mathbb{N}} \sup _{(0, T) \times \mathbb{T}}\left\{\rho^{n}(t, x), 1 / \rho^{n}(t, x)\right\} \leqslant C_{0},
$$

for some constant $C_{0}$.

Thanks to (23)-(24) we have the following bounds:

- $\rho^{n}$ is bounded in $L^{\infty}((0, T) \times \mathbb{T})$;

- $\partial_{x} u^{n}$ is bounded in $L^{2}\left(0, T ; L^{2}(\mathbb{T})\right)$;

- $\sqrt{\rho^{n}} u^{n}$ is bounded in $L^{\infty}\left(0, T ; L^{2}(\mathbb{T})\right)$.

We might then combine the above bounds on $\partial_{x} u^{n}$ with (24) to yield:

- $u^{n}$ is bounded in $L^{2}\left(0, T ; H^{1}(\mathbb{T})\right)$;

- $z^{n}:=\mu^{n} \partial_{x} u^{n}-p^{n}$ is bounded in $L^{2}((0, T) \times \mathbb{T})$.

Up to the extraction of a subsequence, we obtain that:

- $\rho^{n}$ converges to $\bar{\rho}$ in $L^{\infty}((0, T) \times \mathbb{T})-w *$;

- $u^{n}$ converges to $\bar{u}$ in $L^{2}\left(0, T ; H^{1}(\mathbb{T})\right)-w$, and $L^{2}((0, T) \times \mathbb{T})$;

- $z^{n}$ converges to $\bar{z}$ in $L^{2}((0, T) \times \mathbb{T})-w^{*}$.

The strong convergence of the velocity-fields yields as a classical application of Aubin-Lions lemma and relies on the coupling of bounds on $\partial_{x} u^{n}$ and bounds on $\partial_{t} u^{n}$ (obtained thanks to the momentum equation). Combining the above weak limits entails that:

$$
\begin{aligned}
\partial_{t} \bar{\rho}+\partial_{x}(\bar{\rho} \bar{u}) & =0, \\
\partial_{t}(\bar{\rho} \bar{u})+\partial_{x}\left(\bar{\rho} \bar{u}^{2}\right) & =\partial_{x} \bar{z} .
\end{aligned}
$$

One first critical issue is now to compute $\bar{z}$. Indeed, it is not clear that we may pass to the limit in the constitutive equation since it relies on nonlinear quantities $\left(\mu^{n} \partial_{x} u^{n}\right.$ and $\left.p\left(\rho^{n}\right)\right)$ which are not preserved by weak limits. It is then necessary to identify new quantities which are compact for some strong topology, so that we may rewrite $z^{n}$ in terms of these quantities to pass to the limit combining weak and strong limits.

Part of this problem is also related to passing to the limit in nonlinear quantities of $\rho^{n}$. For this, we already showed that we have to rely on the introduction of Young measures $\bar{\nu}$. A second key-issue is then to write an evolution PDE satisfied by these Young measures. Finally, the system (14)-(15)-(17) is obtained by plugging in this evolution equation that the associated generalized functions $x \mapsto \bar{\nu}(t)_{x} \in \mathcal{C}_{2}$ for $t \in(0, T)$. However, we assume in Theorem 5, that this property holds initially only. Hence, a last key-difficulty in the proof of Theorem 5 is to obtain that the property $x \mapsto \bar{\nu}(t)_{x} \in \mathcal{C}_{2}$ is preserved in the homogenization process. For this, an important ingredient is that we may restrict to velocity-fields such that $\partial_{x} \bar{u} \in L^{1}\left(0, T ; L^{\infty}(\mathbb{T})\right)$. This enables to construct classical solutions to (14)-(15)-(17) and perform a weak-strong uniqueness argument on the evolution equations satisfied by the Young measures. 


\section{TEMPERATURE-DEPENDENT MODELS}

In this last section, we proceed with temperature-dependent models. First, we detail the model under consideration (corresponding to steps (1) and (2)). Second, we provide formal estimates satisfied by reasonable solutions and show then which multiphase model the method we described previously would enable to derive. We remain in the $1 \mathrm{D}$ case throughout this section.

\subsection{Microscopic model}

We consider here a mixture of two perfect heat-conductive fluids. We denote $\left(\rho_{+}, u_{+}, p_{+}, \mu_{+}, e_{+}, \theta_{+}\right)$(resp $\left.\left(\rho_{-}, u_{-}, p_{-}, \mu_{-}, e_{-}, \theta_{-}\right)\right)$the density/velocity/pressure/viscosity/internal energy/temperature of phase + (resp. phase - ). In both phases $k=+,-$ these unknowns satisfy the equations of a perfect heat-conductive gas (among the numerous references, see $[10,11]$ ):

$$
\begin{aligned}
& \partial_{t} \rho_{k}+\partial_{x}\left(\rho_{k} u_{k}\right)=0, \\
& \partial_{t}\left(\rho_{k} u_{k}\right)+\partial_{x}\left(\rho_{k} u_{k}^{2}\right)=\partial_{x}\left(\mu_{k} \partial_{x} u_{k}-p_{k}\right), \\
& \partial_{t}\left(\rho_{k} e_{k}\right)+\partial_{x}\left(\rho_{k} u_{k} e_{k}\right)=\partial_{x}\left(\kappa_{k} \partial_{x} \theta_{k}\right)+\left(\mu_{k} \partial_{x} u_{k}-p_{k}\right) \partial_{x} u_{k} .
\end{aligned}
$$

We complement the system with the constitutive equations:

$$
\mu_{k}=\mu_{k}(\rho), \quad p_{k}=c_{P, k} \rho_{k} \theta_{k}, \quad e_{k}=c_{V, k} \theta_{k} .
$$

Here we introduced the thermal capacities $c_{V, k}$ and $c_{P, k}$ and the heat conductivity $\kappa_{k}$. All these parameters are assumed constant (but depending on the phase) and strictly positive. We may rewrite the last equation:

$$
\partial_{t}\left(q_{k} \theta_{k}\right)+\partial_{x}\left(q_{k} u_{k} \theta_{k}\right)=\partial_{x}\left(\kappa_{k} \partial_{x} \theta_{k}\right)+\left(\mu_{k} \partial_{x} u_{k}-p_{k}\right) \partial_{x} u_{k},
$$

where $q_{k}=c_{V, k} \rho_{k}$. One good feature of this new unknown is that, since $c_{V, k}$ is assumed constant (in each phase), there holds:

$$
\partial_{t} q_{k}+\partial_{x}\left(q_{k} u_{k}\right)=0
$$

We prescribe now the interface conditions. We again assume that the phases do not slip at their interface which moves with their joint velocity. We also prescribe continuity of

- normal stresses : $\left[\mu_{k} \partial_{x} u_{k}-p_{k}\right]=0$,

- thermal fluxes : $\left[\kappa_{k} \partial_{x} \theta_{k}\right]=0$.

Doing so, the extended unknowns (we recall that we denote $\Omega_{+}$and $\Omega_{-}$the subsets occupied by phase + and - respectively)

$$
\rho=\rho_{+} \mathbf{1}_{\Omega_{+}}+\rho_{-} \mathbf{1}_{\Omega_{-}}, \quad \quad u=u_{+} \mathbf{1}_{\Omega_{+}}+u_{-} \mathbf{1}_{\Omega_{-}}, \quad \theta=\theta_{+} \mathbf{1}_{\Omega_{+}}+\theta_{-} \mathbf{1}_{\Omega_{-}},
$$

satisfy the extended system

$$
\begin{aligned}
& \partial_{t} \rho+\partial_{x}(\rho u)=0, \\
& \partial_{t} q+\partial_{x}(q u)=0 \\
& \partial_{t}(\rho u)+\partial_{x}\left(\rho u^{2}\right)=\partial_{x}\left(\mu \partial_{x} u-p\right), \\
& \partial_{t}(q \theta)+\partial_{x}(q u \theta)=\partial_{x}\left(\kappa \partial_{x} \theta\right)+\left(\mu \partial_{x} u-p\right) \partial_{x} u,
\end{aligned}
$$

with constitutive equations:

$$
\mu=\tilde{\mu}(\rho), \quad q=\tilde{q}(\rho), \quad \kappa=\tilde{\kappa}(\rho), \quad p=\tilde{c}(\rho) \theta .
$$


Here we introduced $\tilde{\mu}, \tilde{q}, \tilde{\kappa}$ and $\tilde{c}$ functions that are given by the same extension process as in the isentropic case. Under the further assumption that $\rho_{+}$and $\rho_{-}$range two non-overlapping intervals $I_{+}$and $I_{-}$respectively, we set:

$$
(\tilde{\mu}, \tilde{q}, \tilde{\kappa}, \tilde{c})(r)= \begin{cases}\left(\mu_{+}(r), c_{V,+} r, \kappa_{+}, c_{P,+} r\right) & \text { if } r \in I_{+} \\ \left(\mu_{-}(r), c_{V,-} r, \kappa_{-}, c_{P,-} r\right) & \text { if } r \in I_{+}\end{cases}
$$

We assume below that these functions are smooth with

$$
\begin{aligned}
& \tilde{\mu} \geqslant \mu_{0}, \quad \tilde{\kappa} \geqslant \kappa_{0}, \\
& z \mapsto \tilde{p}(z) / z, z \mapsto \tilde{q}(z) / z \text { bounded. }
\end{aligned}
$$

These assumptions are not restrictive in case $\rho_{+}$and $\rho_{-}$range two non-overlapping closed intervals. The unknowns of the above system (25)-(26)-(27)-(28)-(29) are $(\rho, u, \theta)$. It is considered on the torus $\mathbb{T}$ (and thus completed with periodic boundary conditions).

\subsection{Fundamental estimates}

We provide here a family of estimates that solutions to (25)-(26)-(27)-(28)-(29) should satisfy. The computations are inspired of [15]. We restrict to small solutions (in a sense we make precise below), but we allow solutions with large density discontinuities. This latter point is fundamental in the derivation of multiphase systems.

So, we consider in this section datas $\left(\rho^{0}, u^{0}, \theta^{0}\right)$ such that

- $\rho^{0} \in L^{\infty}(\mathbb{T})$ is far from zero,

- $u^{0}, \theta^{0} \in H^{1}(\mathbb{T})$ (with $\theta^{0}>0$ ).

We introduce two constants $\left(V_{\rho}^{0}, V_{\theta}^{0}\right)$ such that there holds initially:

$$
\left\|\rho^{0}\right\|_{L^{\infty}}+\left\|1 / \rho^{0}\right\|_{L^{\infty}} \leqslant V_{\rho}^{0}, \quad\left\|u^{0}\right\|_{H^{1}}+\left\|\theta^{0}\right\|_{H^{1}} \leqslant V^{0}
$$

(we drop the symbol $\mathbb{T}$ in norm notations for legibility). Then, we introduce $M^{0}$ and we prove that, on some time-interval $\left(0, T_{0}\right)$ a solution should satisfy:

$$
\begin{gathered}
\|\rho(t, \cdot)\|_{L^{\infty}}+\|1 / \rho(t, \cdot)\|_{L^{\infty}} \leqslant M^{0}, \quad\|u(t, \cdot)\|_{H^{1}}+\|\theta(t, \cdot)\|_{H^{1}} \leqslant M^{0}, \\
\int_{0}^{t}\left[\left\|\mu \partial_{x} u-p\right\|_{H^{1}}^{2}+\left\|\kappa \partial_{x} \theta\right\|_{H^{1}}^{2}\right] \leqslant\left|M^{0}\right|^{2} .
\end{gathered}
$$

For technical reasons, we have to introduce a constant $\mathcal{E}^{0}$ and restrict to solutions such that

$$
\sup _{(0, T)}\left[\int_{\mathbb{T}} \mu\left|\partial_{x} u-p / \mu\right|^{2}+\int_{\mathbb{T}} \kappa\left|\partial_{x} \theta\right|^{2}\right] \leqslant\left|\mathcal{E}^{0}\right|^{2}
$$

We emphasize that, to obtain (33) we will have to assume that initially

$$
\int_{\mathbb{T}} \mu^{0}\left|\partial_{x} u^{0}-p^{0} / \mu^{0}\right|^{2}+\int_{\mathbb{T}} \kappa^{0}\left|\partial_{x} \theta^{0}\right|^{2}<<\left|\mathcal{E}^{0}\right|^{2}<<\left|M^{0}\right|^{2},
$$

where $\mu^{0}=\tilde{\mu}\left(\rho^{0}\right), \kappa^{0}=\tilde{\kappa}\left(\rho^{0}\right)$ and $p^{0}=\tilde{c}\left(\rho^{0}\right) \theta^{0}$. Thus the estimates we compute below are only valid for well-prepared initial data.

The proof is based on a standard continuation principle: we assume that the large inequalities (31)-(32)-(33) hold on some interval $(0, T)$ and we prove that, as long as $T<T_{0}$ the strict inequality is actually true. A full analytical justification would then be completed thanks to the construction of a sufficiently smooth local 
existence theory. We emphasize that there are three quantities to be fixed with the following estimates : $\mathcal{E}^{0}, M^{0}$ and $T_{0}$. In order that there is no contradiction, we fix $\mathcal{E}^{0}$ and then we compute restrictions on $M^{0}$ (which will have to be sufficiently large) and restrictions on $T_{0}$ (which will have to be sufficiently small).

So, from now on, let assume that we have initial data $\left(\rho^{0}, u^{0}, \theta^{0}\right)$ such that (30) holds true and that we are able to construct a solution $(\rho, u, \theta)$ on $(0, T)$ such that (31)-(32)-(33) hold true.

Preliminaries. We introduce from now on two notations $V^{\infty}$ and $V_{\rho}^{\infty}$. These notations stand for constants which depend only on $M^{0}$ and $V_{\rho}^{0}$ respectively. We forget about dependencies on parameters of the system such as $\tilde{\mu}, \tilde{c} \ldots$ The involved constants may vary between lines. More precisely, when an inequality with such a constant is written, it must be understood as "There exists a constant $V^{\infty} / V_{\rho}^{\infty}$ depending only on $M^{0} / V_{\rho}^{0}$ such that the inequality holds true." We also use the symbol $K$ for constants which depend on the parameters of the problem only (these constants are typically related to Sobolev embeddings). Note that, with these conventions, when a constant $V^{\infty} / V_{\rho}^{\infty}$ and a constant $K$ should be involved together, we can drop the constant $K$.

First, we note that, by a standard embedding arguments, inequality (33) implies:

$$
\int_{0}^{T}\left\|\mu \partial_{x} u-p\right\|_{L^{\infty}}^{2} \leqslant\left|M^{0}\right|^{2}
$$

Since $p$ is a smooth function of $\rho$ and $\theta$ we have also that $\|p(t, \cdot)\|_{\infty} \leqslant V^{\infty}$. Consequently, we obtain that (for $T<1)$, on $(0, T)$ :

$$
\int_{0}^{t}\left\|\partial_{x} u\right\|_{L^{\infty}}^{2} \leqslant\left|V^{\infty}\right|^{2}
$$

We forget here the factor $1 /\left|\mu^{0}\right|^{2}$ on the right-hand side which is related to the data of the system only.

Density estimate. We note that $\rho$ satisfies a transport equation:

$$
\partial_{t} \rho+u \partial_{x} \rho=-\rho \partial_{x} u
$$

Classically (see [2] for instance), we have then (applying (34)):

$$
\begin{aligned}
\|\rho(t, \cdot)\|_{L^{\infty}}+\|1 / \rho(t, \cdot)\|_{L^{\infty}} & \leqslant V_{\rho}^{0} \exp \left(\int_{0}^{t}\left\|\partial_{x} u\right\|_{L^{\infty}}\right) \\
& \leqslant V_{\rho}^{0} \exp \left(\sqrt{t} V^{\infty}\right)
\end{aligned}
$$

Up to assuming that $M^{0}>2 V_{\rho}^{0}$ and $\sqrt{T}<\ln (2) / \ln \left(V^{\infty}+1\right)$ we obtain the first part of (31) and even the finer:

$$
\|\rho(t, \cdot)\|_{L^{\infty}}+\|1 / \rho(t, \cdot)\|_{L^{\infty}} \leqslant 2 V_{\rho}^{0} .
$$

As a consequence, we can assume below that, under the above restriction on $T$, given functions $\beta \in C^{1}((0, \infty))$ we have

$$
|\beta(\rho(t, x))| \leqslant V_{\rho}^{\infty} \quad \text { on }(0, T) \times \mathbb{T} .
$$

Of course, this inequality is not true for all $\beta \in C^{1}((0, \infty))$. But, only a finite number of such functions $\beta$ is involved in the following computations. 
Integrability estimates. Now, we multiply the momentum equation (27) with $u$. This yields:

$$
\frac{\mathrm{d}}{\mathrm{d} t}\left[\int_{\mathbb{T}} \rho \frac{|u|^{2}}{2}\right]+\int_{\mathbb{T}} \mu\left|\partial_{x} u\right|^{2}=\int_{\mathbb{T}} \tilde{c}(\rho) \theta \partial_{x} u
$$

We control here the right-hand side:

$$
|R H S| \leqslant\|\tilde{c}(\rho)\|_{L^{\infty}}\|u\|_{H^{1}}\|\theta\|_{H^{1}} \leqslant V_{\rho}^{\infty}\left|M^{0}\right|^{2} .
$$

Hence, for $T$ sufficiently small w.r.t. $V_{\rho}^{\infty}$ and $M^{0}$, we have, on $(0, T)$ :

$$
\left|\int_{0}^{T} R H S\right| \leqslant V_{\rho}^{\infty}\left|M^{0}\right|^{2} T \leqslant 1,
$$

and consequently:

or

$$
\int_{\mathbb{T}} \rho(t, x) \frac{|u(t, x)|^{2}}{2} \leqslant \int_{\mathbb{T}} \rho^{0}(x) \frac{\left|u^{0}(x)\right|^{2}}{2}+1
$$

$$
\int_{\mathbb{T}}|u(t, x)|^{2} \leqslant 2 V_{\rho}^{\infty}\left[\int_{\mathbb{T}} \rho^{0}(x) \frac{\left|u^{0}(x)\right|^{2}}{2}+1\right] .
$$

Thus, we require $\left|M^{0}\right|^{2} / 4$ to be strictly larger than the right-hand side of this inequality in order that we obtain:

$$
\|u(t, \cdot)\|_{L^{2}}<\frac{M^{0}}{2}, \quad \text { on }(0, T) .
$$

Similarly, we multiply the internal energy equation (28) with $\theta$. This yields:

$$
\frac{\mathrm{d}}{\mathrm{d} t}\left[\int_{\mathbb{T}} q \frac{|\theta|^{2}}{2}\right]+\int_{\mathbb{T}} \kappa\left|\partial_{x} \theta\right|^{2}=\int_{\mathbb{T}} \mu\left|\partial_{x} u\right|^{2} \theta-\int_{\mathbb{T}} \tilde{c}(\rho) \partial_{x} u|\theta|^{2}
$$

We control the right-hand side as follows, with the $L^{\infty}$ bounds on $\mu, p$

$$
\begin{aligned}
R H S & \leqslant K\left[\|\mu\|_{L^{\infty}}\|u\|_{H^{1}}^{2}\|\theta\|_{H^{1}}+\|\tilde{c}\|_{L^{\infty}}\|u\|_{H^{1}}\|\theta\|_{H^{1}}^{2}\right] \\
& \leqslant V_{\rho}^{\infty}\left|M^{0}\right|^{3} .
\end{aligned}
$$

Hence, for $T$ sufficiently small w.r.t. $M^{0}$ and $V_{\rho}^{\infty}$, we obtain that, on $(0, T)$

$$
\int_{\mathbb{T}} q(t, x) \frac{|\theta(t, x)|^{2}}{2} \mathrm{~d} x+\int_{0}^{t} \int_{\mathbb{T}} \kappa\left|\partial_{x} \theta\right|^{2} \leqslant \int_{\mathbb{T}} \tilde{q}\left(\rho^{0}(x)\right) \frac{\left|\theta^{0}(x)\right|^{2}}{2} \mathrm{~d} x+1,
$$

and

$$
\int_{\mathbb{T}}|\theta(t, x)|^{2} \mathrm{~d} x \leqslant 2 V_{\rho}^{\infty}\left[\int_{\mathbb{T}} \tilde{q}\left(\rho^{0}(x)\right) \frac{\left|\theta^{0}(x)\right|^{2}}{2} \mathrm{~d} x+1\right] .
$$

Up to assume that $\left|M^{0}\right|^{2} / 4$ is larger than the right-hand side of this latter inequality, we obtain again:

$$
\|\theta\|_{L^{2}} \leqslant \frac{M^{0}}{2}, \quad \text { on }(0, T)
$$


Further regularity estimates. We want now to complete the computation of (32) and obtain (33). We focus on (33) since (32) is a consequence of (33) together with (37)-(38) up to the condition that $M^{0}$ is sufficiently large w.r.t. $V_{\rho}^{\infty}$ and $\mathcal{E}^{0}$.

To proceed with the computation of $(33)$ we introduce some notations. In the $L^{2}(\mathbb{T})$-space, we denote $\mathbb{P}_{0}$ the orthogonal projector on the subspace of mean-free functions $L_{0}^{2}(\mathbb{T})$. We also denote $\mathbb{E}_{0}$ the mean operator and $\partial_{x}^{-1}$ the operator which maps an $L_{0}^{2}(\mathbb{T})$-function to its mean-free primitive in $H^{1}(\mathbb{T})$. It is straightforward that $\partial_{x}^{-1}$ is a bounded mapping $H^{m}(\mathbb{T}) \cap L_{0}^{2}(\mathbb{T}) \rightarrow H^{m+1}(\mathbb{T}) \cap L_{0}^{2}(\mathbb{T})$. It can be extended to negative Sobolev-spaces (i.e. $H^{-m}(\mathbb{T})$ the dual of $\left.H^{m}(\mathbb{T}) \cap L_{0}^{2}(\mathbb{T})\right)$. We remark also that $\mathbb{P}_{0}[v]=v-\mathbb{E}_{0}[v]$.

So, we multiply the momentum equation (27) with $v=\partial_{x}^{-1}\left(\partial_{t} \mathbb{P}_{0}\left(\partial_{x} u-p / \mu\right)\right)$. We obtain:

$$
\int_{\mathbb{T}} \rho\left(\partial_{t} u+u \partial_{x} u\right) v=\int_{\mathbb{T}} \partial_{x}\left(\mu \partial_{x} u-p\right) v
$$

On the right-hand side, we have:

$$
\begin{aligned}
R H S= & \int_{\mathbb{T}} \partial_{x}\left(\mu \partial_{x} u-p\right) v \\
= & -\int_{\mathbb{T}}\left(\mu \partial_{x} u-p\right) \partial_{t}\left[\left(\partial_{x} u-p / \mu\right)+\mathbb{E}_{0}(p / \mu)\right] \\
= & -\int_{\mathbb{T}} \frac{\mu}{2} \partial_{t}\left[\left|\partial_{x} u-p / \mu\right|^{2}\right]-\frac{\mathrm{d}}{\mathrm{d} t}\left[\frac{1}{2 \pi} \int_{\mathbb{T}} \frac{p}{\mu}\right] \int_{\mathbb{T}}\left(\mu \partial_{x} u-p\right) \\
= & -\frac{1}{2} \frac{\mathrm{d}}{\mathrm{d} t}\left[\int_{\mathbb{T}} \mu\left|\partial_{x} u-\frac{p}{\mu}\right|^{2}\right]+\int_{\mathbb{T}} \partial_{t} \mu\left|\partial_{x} u-\frac{p}{\mu}\right|^{2} \\
& -\frac{1}{2 \pi} \frac{\mathrm{d}}{\mathrm{d} t}\left[\int_{\mathbb{T}} \frac{p}{\mu}\right] \int_{\mathbb{T}}\left(\mu \partial_{x} u-p\right) .
\end{aligned}
$$

On the left-hand side, there holds

$$
\begin{aligned}
L H S & =\int_{\mathbb{T}} \rho\left(\partial_{t} u+u \partial_{x} u\right) \partial_{t} u-\int_{\mathbb{T}} \rho\left(\partial_{t} u+u \partial_{x} u\right) \partial_{x}^{-1}\left(\mathbb{P}_{0} \partial_{t}(p / \mu)\right) \\
& =\int_{\mathbb{T}} \rho\left|\partial_{t} u+u \partial_{x} u\right|^{2}-\int_{\mathbb{T}} \rho\left(\partial_{t} u+u \partial_{x} u\right)\left(u \partial_{x} u+\partial_{x}^{-1}\left(\mathbb{P}_{0} \partial_{t}(p / \mu)\right)\right) .
\end{aligned}
$$

Consequently, we rewrite our estimate:

$$
\frac{1}{2} \frac{\mathrm{d}}{\mathrm{d} t}\left[\int_{\mathbb{T}} \mu\left|\partial_{x} u-\frac{p}{\mu}\right|^{2}\right]+\int_{\mathbb{T}} \frac{1}{\rho}\left|\partial_{x}\left(\mu \partial_{x} u-p\right)\right|^{2}=-I_{1}-I_{2}+I_{3},
$$

where

$$
\begin{aligned}
I_{1} & =\int_{\mathbb{T}} \partial_{t}\left[\frac{1}{\mu}\right]\left|\mu \partial_{x} u-p\right|^{2}, \\
I_{2} & =\frac{1}{2 \pi} \frac{\mathrm{d}}{\mathrm{d} t}\left[\int_{\mathbb{T}} \frac{p}{\mu}\right] \int_{\mathbb{T}}\left(\mu \partial_{x} u-p\right), \\
I_{3} & =\int_{\mathbb{T}} \rho\left(\partial_{t} u+u \partial_{x} u\right)\left(u \partial_{x} u+\partial_{x}^{-1}\left(\mathbb{P}_{0} \partial_{t}(p / \mu)\right)\right) .
\end{aligned}
$$


To compute these integrals, we remark that, for arbitrary $\beta \in C^{1}((0, \infty))$ we may multiply the continuity equation $(25) \beta^{\prime}(\rho)$. This yields:

$$
\partial_{t} \beta(\rho)+\partial_{x}(\beta(\rho) u)+\left(\beta^{\prime}(\rho) \rho-\beta(\rho)\right) \partial_{x} u=0 .
$$

Replacing $\beta$ with $\beta_{0}=1 / \tilde{\mu}$ in this last equation, we obtain:

$$
\partial_{t}\left[\frac{1}{\mu}\right]=-\partial_{x}\left(\beta_{0}(\rho) u\right)-\left(\beta_{0}^{\prime}(\rho) \rho-\beta_{0}(\rho)\right) \partial_{x} u
$$

In particular, there holds

$$
\begin{aligned}
I_{1}:=\int_{\mathbb{T}} \partial_{t}\left[\frac{1}{\mu}\right]\left|\mu \partial_{x} u-p\right|^{2}= & 2 \int_{\mathbb{T}} \beta_{0}(\rho) u\left(\mu \partial_{x} u-p\right) \partial_{x}\left(\mu \partial_{x} u-p\right) \\
& -\int_{\mathbb{T}}\left(\beta_{0}^{\prime}(\rho) \rho-\beta_{0}(\rho)\right) \partial_{x} u\left|\mu \partial_{x} u-p\right|^{2}
\end{aligned}
$$

Concerning the first term on the right-hand side, we have, combining standard Young, Hölder and Sobolev inequalities:

$$
\begin{aligned}
\left|\int_{\mathbb{T}} \beta_{0}(\rho) u\left(\mu \partial_{x} u-p\right) \partial_{x}\left(\mu \partial_{x} u-p\right)\right| & \leqslant 4\left\|\beta_{0}(\rho) u\right\|_{L^{\infty}}^{2} \int_{\mathbb{T}} \mu^{2} \rho\left|\partial_{x} u-p / \mu\right|^{2}+\frac{1}{4} \int_{\mathbb{T}} \frac{1}{\rho}\left|\partial_{x}\left(\mu \partial_{x} u-p\right)\right|^{2} \\
& \leqslant V_{\rho}^{\infty}\left|M^{0}\right|^{2} \int_{\mathbb{T}} \mu\left|\partial_{x} u-p / \mu\right|^{2}+\frac{1}{4} \int_{\mathbb{T}} \frac{1}{\rho}\left|\partial_{x}\left(\mu \partial_{x} u-p\right)\right|^{2} .
\end{aligned}
$$

Remarking that $\left|\left(\beta_{0}^{\prime}(\rho) \rho-\beta_{0}(\rho)\right) / \tilde{\mu}(\rho)\right| \leqslant V_{\rho}^{\infty}$ on $(0, T)$ we bound:

$$
\left|\int_{\mathbb{T}}\left(\beta_{0}^{\prime}(\rho) \rho-\beta_{0}(\rho)\right) \partial_{x} u\right| \partial_{x} u-\left.\frac{p}{\mu}\right|^{2}\left|\leqslant V_{\rho}^{\infty}\left\|\partial_{x} u\right\|_{L^{\infty}} \int_{\mathbb{T}} \mu\right| \partial_{x} u-\left.\frac{p}{\mu}\right|^{2},
$$

and finally:

$$
\left|I_{1}\right| \leqslant V_{\rho}^{\infty}\left(\left|M^{0}\right|^{2}+\left\|\partial_{x} u\right\|_{L^{\infty}}\right) \int_{\mathbb{T}} \mu\left|\partial_{x} u-\frac{p}{\mu}\right|^{2}+\frac{1}{4} \int_{\mathbb{T}} \frac{1}{\rho}\left|\partial_{x}\left(\mu \partial_{x} u-p\right)\right|^{2} .
$$

Let plug then $\beta_{1}=\tilde{c} / \tilde{\mu}$ into (40). We obtain:

$$
\partial_{t} \beta_{1}(\rho)+\partial_{x}\left(\beta_{1}(\rho) u\right)=-\left(\beta_{1}^{\prime}(\rho) \rho-\beta_{1}(\rho)\right) \partial_{x} u
$$

and, combining with (28), we obtain:

$$
\begin{aligned}
\partial_{t}\left[\frac{p}{\mu}\right] & =\partial_{t}\left(\beta_{1}(\rho) \theta\right) \\
& =-\partial_{x}\left(\beta_{1}(\rho) u \theta\right)-\left(\beta_{1}^{\prime}(\rho) \rho-\beta_{1}(\rho)\right) \theta \partial_{x} u+\frac{\beta_{1}(\rho)}{\sqrt{q(\rho)}}\left[\sqrt{q(\rho)}\left(\partial_{t} \theta+u \partial_{x} \theta\right)\right] .
\end{aligned}
$$

As a first consequence, there holds

$$
\frac{\mathrm{d}}{\mathrm{d} t} \int_{\mathbb{T}} \frac{p}{\mu}=-\int_{\mathbb{T}}\left(\beta_{1}^{\prime}(\rho) \rho-\beta_{1}(\rho)\right) \theta \partial_{x} u+\int_{\mathbb{T}} \frac{\beta_{1}(\rho)}{\sqrt{q(\rho)}}\left[\sqrt{q(\rho)}\left(\partial_{t} \theta+u \partial_{x} \theta\right)\right]
$$


Since all functions of $\rho$ may be bounded by $V_{\rho}^{\infty}$ on $(0, T)$ we conclude that

$$
\left|\frac{\mathrm{d}}{\mathrm{d} t} \int_{\mathbb{T}} \frac{p}{\mu}\right| \leqslant V_{\rho}^{\infty}\left[\|\theta\|_{L^{2}}\left\|\partial_{x} u\right\|_{L^{2}}+\left\|\sqrt{q(\rho)}\left(\partial_{t} \theta+u \partial_{x} \theta\right)\right\|_{L^{2}}\right],
$$

and

$$
\begin{aligned}
\left|I_{2}\right| & \leqslant V_{\rho}^{\infty}\left[\|\theta\|_{L^{2}}\left\|\partial_{x} u\right\|_{L^{2}}+\left\|\sqrt{q(\rho)}\left(\partial_{t} \theta+u \partial_{x} \theta\right)\right\|_{L^{2}}\right]\left[\left\|\partial_{x} u\right\|_{L^{2}}+\|\theta\|_{L^{2}}\right] \\
& \leqslant V_{\rho}^{\infty}\left[\left|M^{0}\right|^{2}+\left|M^{0}\right|^{3}+\left\|\sqrt{q(\rho)}\left(\partial_{t} \theta+u \partial_{x} \theta\right)\right\|_{L^{2}}^{2}\right] .
\end{aligned}
$$

Secondly, there holds:

$$
\begin{aligned}
\left.\partial_{x}^{-1}\left(\mathbb{P}_{0} \partial_{t}(p / \mu)\right)\right)= & \mathbb{P}_{0}\left[\beta_{1}(\rho) u\right]-\partial_{x}^{-1} \mathbb{P}_{0}\left[\left(\beta_{1}^{\prime}(\rho) \rho-\beta_{1}(\rho)\right) \theta \partial_{x} u\right] \\
& +\partial_{x}^{-1} \mathbb{P}_{0}\left[\frac{\beta_{1}(\rho)}{\sqrt{q(\rho)}}\left[\sqrt{q(\rho)}\left(\partial_{t} \theta+u \partial_{x} \theta\right)\right]\right]
\end{aligned}
$$

Since $\partial_{x}^{-1}$ and $\mathbb{P}_{0}$ are bounded mappings, we proceed with:

$$
\begin{aligned}
\left.\| \partial_{x}^{-1}\left(\mathbb{P}_{0} \partial_{t}(p / \mu)\right)\right) \|_{L^{2}} \leqslant & \left\|\beta_{1}(\rho) u\right\|_{L^{2}}+\left\|\left(\beta_{1}^{\prime}(\rho) \rho-\beta_{1}(\rho)\right) \theta \partial_{x} u\right\|_{L^{2}} \\
& +\left\|\frac{\beta_{1}(\rho)}{\sqrt{q(\rho)}}\left[\sqrt{q(\rho)}\left(\partial_{t} \theta+u \partial_{x} \theta\right)\right]\right\|_{L^{2}}, \\
\leqslant & V_{\rho}^{\infty}\left[\|u\|_{H^{1}}+\|\theta\|_{H^{1}}\left\|\partial_{x} u\right\|_{L^{2}}+\left\|\sqrt{q(\rho)}\left(\partial_{t} \theta+u \partial_{x} \theta\right)\right\|_{L^{2}}\right] .
\end{aligned}
$$

Consequently:

$$
\begin{aligned}
\left|I_{3}\right| \leqslant & \left.\frac{1}{4} \int_{\mathbb{T}} \rho\left|\partial_{t} u+u \partial_{x} u\right|^{2}+\int_{\mathbb{T}} \rho \mid u \partial_{x} u+\partial_{x}^{-1}\left(\mathbb{P}_{0} \partial_{t}(p / \mu)\right)\right)\left.\right|^{2} \\
\leqslant & \frac{1}{4} \int_{\mathbb{T}} \rho\left|\partial_{t} u+u \partial_{x} u\right|^{2} \\
& +V_{\rho}^{\infty}\left[\|u\|_{H^{1}}^{4}+\left[\|u\|_{H^{1}}+\|\theta\|_{H^{1}}\left\|\partial_{x} u\right\|_{L^{2}}+\left\|\sqrt{q(\rho)}\left(\partial_{t} \theta+u \partial_{x} \theta\right)\right\|_{L^{2}}\right]^{2}\right] \\
\leqslant & \frac{1}{4} \int_{\mathbb{T}} \rho\left|\partial_{t} u+u \partial_{x} u\right|^{2}+V_{\rho}^{\infty}\left[\left|M^{0}\right|^{2}\left(1+\left|M^{0}\right|^{2}\right)+\left\|\sqrt{q(\rho)}\left(\partial_{t} \theta+u \partial_{x} \theta\right)\right\|_{L^{2}}^{2}\right] .
\end{aligned}
$$

Introducing the computations of $I_{1}, I_{2}, I_{3}$ into (39) we obtain:

$$
\begin{aligned}
\frac{1}{2} \frac{\mathrm{d}}{\mathrm{d} t}\left[\int_{\mathbb{T}} \mu\left|\partial_{x} u-\frac{p}{\mu}\right|^{2}\right]+\frac{1}{2} \int_{\mathbb{T}} \frac{1}{\rho}\left|\partial_{x}\left(\mu \partial_{x} u-p\right)\right|^{2} & \\
\leqslant & V_{\rho}^{\infty}\left\{\left(\left|M^{0}\right|^{2}+\left\|\partial_{x} u\right\|_{L^{\infty}}\right) \int_{\mathbb{T}} \mu\left|\partial_{x} u-\frac{p}{\mu}\right|^{2}\right. \\
& \left.+\left[\left|M^{0}\right|^{2}\left(1+\left|M^{0}\right|^{2}\right)+\left\|\sqrt{q(\rho)}\left(\partial_{t} \theta+u \partial_{x} \theta\right)\right\|_{L^{2}}^{2}\right]\right\} .
\end{aligned}
$$


Similarly, we multiply the internal energy equation with $\partial_{t} \theta$. This yields:

$$
\int_{\mathbb{T}} q\left(\partial_{t} \theta+u \partial_{x} \theta\right) \partial_{t} \theta=\int_{\mathbb{T}} \partial_{x}\left(\kappa \partial_{x} \theta\right) \partial_{t} \theta+\int_{\mathbb{T}}\left(\mu \partial_{x} u-p\right) \partial_{x} u \partial_{t} \theta
$$

On the right-hand side, the first term reads:

$$
\int_{\mathbb{T}} \partial_{x}\left(\kappa \partial_{x} \theta\right) \partial_{t} \theta=-\frac{\mathrm{d}}{\mathrm{d} t} \int_{\mathbb{T}} \kappa \frac{\left|\partial_{x} \theta\right|^{2}}{2}-\int_{\mathbb{T}} \partial_{t}\left[\frac{1}{\kappa}\right] \frac{\left|\kappa \partial_{x} \theta\right|^{2}}{2} .
$$

Let denote by $R_{1}$ the second integral appearing on the right-hand side of this last identity. Setting $\beta_{2}=1 / \tilde{\kappa}$ and proceeding as for the integral $I_{1}$ above, we compute that for arbitrary $\varepsilon>0$ there holds

$$
\left|R_{1}\right| \leqslant \frac{V_{\rho}^{\infty}}{\varepsilon}\left[\left|M^{0}\right|^{2}+\left\|\partial_{x} u\right\|_{L^{\infty}}\right] \int_{\mathbb{T}} \kappa \frac{\left|\partial_{x} \theta\right|^{2}}{2}+\varepsilon \int_{\mathbb{T}}\left|\partial_{x}\left(\kappa \partial_{x} \theta\right)\right|^{2} .
$$

At this point, we remark that

$$
\partial_{x}\left(\kappa \partial_{x} \theta\right)=q\left(\partial_{t} \theta+u \partial_{x} \theta\right)-\left(\mu \partial_{x} u-p\right) \partial_{x} u
$$

so that

$$
\int_{\mathbb{T}}\left|\partial_{x}\left(\kappa \partial_{x} \theta\right)\right|^{2} \leqslant 2 V_{\rho}^{\infty} \int_{\mathbb{T}} q\left|\partial_{t} \theta+u \partial_{x} \theta\right|^{2}+2 V_{\rho}^{\infty}\left\|\partial_{x} u\right\|_{L^{\infty}}^{2} \int_{\mathbb{T}} \mu\left|\partial_{x} u-p / \mu\right|^{2} .
$$

We choose then $\varepsilon$ sufficiently small and conclude that:

$$
\begin{aligned}
\left|R_{1}\right| \leqslant & V_{\rho}^{\infty}\left[\left|M^{0}\right|^{2}+\left\|\partial_{x} u\right\|_{\left.L^{\infty}\right]} \int_{\mathbb{T}} \kappa \frac{\left|\partial_{x} \theta\right|^{2}}{2}\right. \\
& +V_{\rho}^{\infty}\left\|\partial_{x} u\right\|_{L^{\infty}}^{2} \int_{\mathbb{T}} \mu\left|\partial_{x} u-p / \mu\right|^{2}+\frac{1}{4} \int_{\mathbb{T}} q\left|\partial_{t} \theta+u \partial_{x} \theta\right|^{2} .
\end{aligned}
$$

As for the second term on the right-hand side of (42) (denoted $R_{2}$ ), we have:

$$
R_{2}:=\int_{\mathbb{T}}\left(\mu \partial_{x} u-p\right) \partial_{x} u \partial_{t} \theta=\int_{\mathbb{T}}\left(\mu \partial_{x} u-p\right) \partial_{x} u\left(\partial_{t} \theta+u \partial_{x} \theta\right)-\int_{\mathbb{T}}\left(\mu \partial_{x} u-p\right) u \partial_{x} u \partial_{x} \theta,
$$

so that:

$$
\begin{aligned}
\left|R_{2}\right| \leqslant & V_{\rho}^{\infty}\left\|\partial_{x} u\right\|_{L^{\infty}}^{2} \int_{\mathbb{T}} \mu\left|\partial_{x} u-p / \mu\right|^{2} \\
& +V_{\rho}^{\infty} M^{0}\left\|\partial_{x} u\right\|_{L^{\infty}}\left[\int_{\mathbb{T}} \mu\left|\partial_{x} u-p / \mu\right|^{2}+\int_{\mathbb{T}} \kappa\left|\partial_{x} \theta\right|^{2}\right] \\
& +\frac{1}{4} \int_{\mathbb{T}} q\left|\partial_{t} \theta+u \partial_{x} \theta\right|^{2}, \\
\leqslant & V_{\rho}^{\infty}\left(M^{0}\left\|\partial_{x} u\right\|_{L^{\infty}}+\left\|\partial_{x} u\right\|_{L^{\infty}}^{2}\right)\left[\int_{\mathbb{T}} \mu\left|\partial_{x} u-p / \mu\right|^{2}+\int_{\mathbb{T}} \kappa\left|\partial_{x} \theta\right|^{2}\right] \\
& +\frac{1}{4} \int_{\mathbb{T}} q\left|\partial_{t} \theta+u \partial_{x} \theta\right|^{2} .
\end{aligned}
$$

It remains to rewrite the left-hand side of (42)

$$
L H S=\int_{\mathbb{T}} q\left|\partial_{t} \theta+u \partial_{x} \theta\right|^{2}-L_{1},
$$


with

$$
\left|L_{1}\right|=\left|\int_{\mathbb{T}} q\left(\partial_{t} \theta+u \partial_{x} \theta\right) u \partial_{x} \theta\right| \leqslant \frac{1}{8} \int_{\mathbb{T}} q\left|\partial_{t} \theta+u \partial_{x} \theta\right|^{2}+V_{\rho}^{\infty}\|u\|_{H^{1}}^{2}\|\theta\|_{H^{1}}^{2}
$$

Combining the above computations of $R_{1}, R_{2}, L_{1}$ into (42) we conclude that

$$
\begin{aligned}
\frac{\mathrm{d}}{\mathrm{d} t} \int_{\mathbb{T}} \kappa \frac{\left|\partial_{x} \theta\right|^{2}}{2}+\frac{1}{8} \int_{\mathbb{T}} q \mid \partial_{t} \theta+ & \left.u \partial_{x} \theta\right|^{2} \\
& \leqslant V_{\rho}^{\infty}\left[1+M^{0}+\left\|\partial_{x} u\right\|_{L^{\infty}}\right]^{2}\left[\int_{\mathbb{T}} \mu\left|\partial_{x} u-p / \mu\right|^{2}+\int_{\mathbb{T}} \kappa\left|\partial_{x} \theta\right|^{2}\right]+V_{\rho}^{\infty}\left|M^{0}\right|^{4}
\end{aligned}
$$

At this point, we remark that we obtained (41) and (43) with a priori distinct constants $V_{\rho}^{\infty}$. However, we may assume that both constants are equal up to take the maximum of the two values that we fix from now on. Combining then (41) with $16 V_{\rho}^{\infty} \times(43)$ and denoting

$$
Y(t)=\frac{1}{2} \int_{\mathbb{T}}\left[\mu\left|\partial_{x} u-p / \mu\right|^{2}+16 V_{\rho}^{\infty} \kappa\left|\partial_{x} \theta\right|^{2}\right],
$$

we obtain:

$$
\frac{\mathrm{d}}{\mathrm{d} t} Y(t) \leqslant K\left(1+V_{\rho}^{\infty}\right) V_{\rho}^{\infty}\left[1+M^{0}+\left\|\partial_{x} u\right\|_{L^{\infty}}\right]^{2} Y(t)+K V_{\rho}^{\infty}\left|M^{0}\right|^{2}\left(1+\left|M^{0}\right|^{2}\right)
$$

Via a standard Gronwall lemma and recalling (34), this yields that

$$
\begin{aligned}
Y(t) & \left.\leqslant\left(Y(0)+t K V_{\rho}^{\infty}\left|M^{0}\right|^{2}\left(1+\left|M^{0}\right|^{2}\right)\right) \exp \left(\int_{0}^{t} K\left(1+V_{\rho}^{\infty}\right) V_{\rho}^{\infty} \mid\left[1+\left|M^{0}\right|+\left\|\partial_{x} u\right\|_{L^{\infty}}\right]^{2}\right)\right) \\
& \leqslant\left(Y(0)+T K V_{\rho}^{\infty}\left|M^{0}\right|^{2}\left(1+\left|M^{0}\right|^{2}\right)\right) \exp \left[K\left(1+V_{\rho}^{\infty}\right) V_{\rho}^{\infty}(1+T)\left(1+\left|M^{0}\right|^{2}\right)\right] .
\end{aligned}
$$

Under the assumptions that $T$ is sufficiently small, and $Y(0)$ sufficiently close to 0 , the above estimate implies that

$$
Y(t)<\frac{1}{16 V_{\rho}^{\infty}}\left|\mathcal{E}^{0}\right|^{2} \quad \text { on }(0, T),
$$

so that we conclude that we have indeed

$$
\sup _{(0, T)}\left[\int_{\mathbb{T}} \mu\left|\partial_{x} u-p / \mu\right|^{2}+\int_{\mathbb{T}} \kappa\left|\partial_{x} \theta\right|^{2}\right] \leqslant\left|\mathcal{E}^{0}\right|^{2}
$$

This concludes the computation of our estimates.

\subsection{Multiphase temperature-dependent system}

To derive the multiphase system corresponding to (25)-(26)-(27)-(28)-(29), we consider now a sequence $\left(\rho^{n}, u^{n}, \theta^{n}\right)_{n \in \mathbb{N}}$ of solution to this system which satisfies $(31)-(32)-(33)$ on $(0, T)$ with $\left(M^{0}, \mathcal{E}^{0}, T\right)$ independent of $n$. We address at first a limit problem and then introducing Young measures and particularly generalized functions in $\mathcal{C}_{2}$ we obtain a bi-fluid sytem.

Derivation of a Navier Stokes-like system. From (31)-(32)-(33), we infer the following bounds:

- $u^{n}$ and $\theta^{n}$ are bounded in $L^{\infty}\left(0, T ; H^{1}(\mathbb{T})\right)$ with time-derivatives $\partial_{t} u^{n}$ and $\partial_{t} \theta^{n}$ bounded in $L^{2}\left(0, T ; L^{2}(\mathbb{T})\right)$;

- $\rho^{n}$ is bounded in $L^{\infty}\left(0, T ; L^{\infty}(\mathbb{T})\right)$;

- $z_{f}^{n}:=\mu^{n} \partial_{x} u^{n}-p^{n}$ and $z_{h}^{n}:=\kappa^{n} \partial_{x} \theta^{n}$ are bounded in $L^{2}\left(0, T ; H^{1}(\mathbb{T})\right)$. 
Here we denote $\mu^{n}=\tilde{\mu}\left(\rho^{n}\right)$ and $\kappa^{n}=\tilde{\kappa}\left(\rho^{n}\right)$ for short. As a first consequence, Ascoli-Arzela like arguments imply that we can extract a subsequence (that we do not relabel) for which:

$$
u^{n} \rightarrow \bar{u}, \quad \theta^{n} \rightarrow \bar{\theta}, \quad \text { in } C\left([0, T] ; L^{2}(\mathbb{T})\right) .
$$

Classical arguments also entail that the fluid and heat fluxes converge weakly:

$$
z_{f}^{n} \rightarrow \bar{z}_{f}, \quad z_{h}^{n} \rightarrow \bar{z}_{h}, \quad \text { in } L^{2}\left(0, T ; H^{1}(\mathbb{T})\right)-w .
$$

Furthermore, we can construct the Young measures $\bar{\nu}(t)$ associated with the sequence of densities $\left(\rho^{n}\right)_{n \in \mathbb{N}}$. Thanks to the uniform bounds on $\rho^{n}$ and $1 / \rho^{n}$, they have compact support in $(0, \infty)$. This entails that for arbitrary $\beta \in C((0, \infty))$ we have

$$
\beta\left(\rho_{n}\right) \rightarrow \bar{\beta}=(t, x) \mapsto\left\langle\bar{\nu}(t)_{x}, \beta\right\rangle \quad \text { in } L^{\infty}\left((0, T) ; L^{\infty}(\mathbb{T})\right)-w * .
$$

All these informations are sufficient to pass to the limit in the fluid and internal-energy equation. Indeed, concerning the energy equation, we first rewrite the $n$-th equation:

$$
\partial_{t}\left(q^{n} \theta^{n}\right)+\partial_{x}\left(q^{n} u^{n} \theta^{n}\right)=\partial_{x} z_{h}^{n}+\partial_{x}\left(z_{f}^{n} u^{n}\right)-\left(\partial_{x} z_{f}^{n}\right) u^{n} .
$$

We can then mix the weak convergences of $q^{n}, z_{f}^{n}$ and the strong convergences of $\theta^{n}, u^{n}$ to obtain in the limit $n \rightarrow \infty$ that:

$$
\partial_{t}(\bar{q} \bar{\theta})+\partial_{x}(\bar{q} \bar{u} \bar{\theta})=\partial_{x} \bar{z}_{h}+\bar{z}_{f} \partial_{x} \bar{u}
$$

(where $\bar{q}(t, x)=\left\langle\bar{\nu}(t)_{x}, \tilde{q}\right\rangle$ ). With comparable arguments, we obtain the limit fluid equation:

$$
\partial_{t}(\bar{\rho} \bar{u})+\partial_{x}\left(\bar{\rho} \bar{u}^{2}\right)=\partial_{x} \bar{z}_{f}
$$

(where $\left.\bar{\rho}(x)=\left\langle\bar{\nu}(t)_{x}, \xi \mapsto \xi\right\rangle\right)$. To complete the system, we thus have to find new constitutive laws which relate $\bar{z}_{f}$ and $\bar{z}_{h}$ to the other unknowns and to write an equation on the Young measures $\bar{\nu}(t) /$ generalized function $x \mapsto \bar{\nu}(t)_{x}$.

Equations on Young measures and multiphase constitutive laws. In order to complete the derivation of the limit system, the main tool is the following compensated compactness result:

Lemma 6. Let $\beta \in C^{1}((0, \infty))$. Then, up to the extraction of a subsequence, there holds:

$$
\beta\left(\rho^{n}\right) z_{f}^{n} \rightarrow \bar{\beta} \bar{z}_{f}, \quad \beta\left(\rho^{n}\right) \theta^{n} \rightarrow \bar{\beta} \bar{\theta}, \quad \beta\left(\rho^{n}\right) z_{h}^{n}-\bar{\beta} \bar{z}_{h}, \quad \text { in } \mathcal{D}^{\prime}((0, T) \times \mathbb{T}) .
$$

Proof. We give a short idea of the proof. More details can be found in [2], see the proof of Lemma 10. The three results consist in considering a function $\beta \in C^{1}((0, \infty))$ and focusing on the convergence of $\beta\left(\rho^{n}\right)$ multiplied by a sequence $z^{n}$ which is bounded in $L^{2}\left(0, T ; H^{1}(\mathbb{T})\right)$. We introduce (we recall that $\mathbb{E}_{0}$ is the mean operator):

$$
w^{n}=\partial_{x}^{-1}\left(\beta\left(\rho^{n}\right)-\mathbb{E}_{0}\left[\beta\left(\rho^{n}\right)\right]\right) .
$$

By computing $\partial_{t} w^{n}$ and $\partial_{t} \mathbb{E}_{0}\left[\beta\left(\rho^{n}\right)\right]$ - as we computed the term $\beta_{1}$ in the previous paragraph - we obtain that (up to the extraction of a subsequence)

$$
\begin{aligned}
& \mathbb{E}_{0}\left[\beta\left(\rho^{n}\right)\right] \rightarrow \mathbb{E}_{0}[\bar{\beta}] \text { in } C([0, T), \\
& w^{n} \rightarrow \bar{w}=\partial_{x}^{-1}\left(\bar{\beta}-\mathbb{E}_{0}[\bar{\beta}]\right) \text { in } C\left([0, T] ; L^{2}(\mathbb{T})\right) .
\end{aligned}
$$


We can then rewrite, for any test-function $\varphi \in C_{c}^{\infty}((0, T) \times(-\pi, \pi))$ that :

$$
\int_{0}^{T} \int_{-\pi}^{\pi} \beta\left(\rho^{n}\right) z^{n} \varphi=\int_{0}^{T} \int_{-\pi}^{\pi} \partial_{x} w^{n} z^{n} \varphi+\int_{0}^{T} \int_{-\pi}^{\pi} \mathbb{E}_{0}\left[\beta\left(\rho^{n}\right)\right] z^{n} \varphi .
$$

Integrating by parts the first term and combining the weak convergence of $z^{n}$ and the strong convergence of $w^{n}, \mathbb{E}_{0}\left[\beta\left(\rho^{n}\right)\right]$, we obtain that

$$
\begin{aligned}
\int_{0}^{T} \int_{-\pi}^{\pi} \beta\left(\rho^{n}\right) z^{n} \varphi & \rightarrow \int_{0}^{T} \int_{-\pi}^{\pi} \partial_{x} \bar{w} \bar{z} \varphi+\int_{0}^{T} \int_{-\pi}^{\pi} \mathbb{E}_{0}[\bar{\beta}] \bar{z} \varphi \\
& \rightarrow \int_{0}^{T} \int_{-\pi}^{\pi} \bar{\beta} \bar{z} \varphi
\end{aligned}
$$

This ends the proof.

To obtain the limit equations on Young measures, we first recall that renormalization theory entails that, for arbitrary $\beta \in C^{1}((0, \infty))$ we have the family of equations:

$$
\partial_{t} \beta\left(\rho^{n}\right)+\partial_{x}\left(\beta\left(\rho^{n}\right) u^{n}\right)+\left(\beta^{\prime}\left(\rho^{n}\right) \rho^{n}-\beta\left(\rho^{n}\right)\right) \partial_{x} u^{n}=0,
$$

that we may rewrite:

$$
\partial_{t} \beta\left(\rho^{n}\right)+\partial_{x}\left(\beta\left(\rho^{n}\right) u^{n}\right)+\frac{\left(\beta^{\prime}\left(\rho^{n}\right) \rho^{n}-\beta\left(\rho_{n}\right)\right)}{\tilde{\mu}\left(\rho^{n}\right)} z_{f}^{n}+\frac{\left(\beta^{\prime}\left(\rho^{n}\right) \rho^{n}-\beta\left(\rho^{n}\right)\right) \tilde{c}\left(\rho^{n}\right)}{\tilde{\mu}\left(\rho^{n}\right)} \theta^{n}=0 .
$$

To pass to the limit in this equation, we apply two items of Lemma 6 which yields that, for arbitrary $\beta \in$ $C^{2}((0, \infty))$ :

$$
\partial_{t} \bar{\beta}+\partial_{x}(\bar{\beta} \bar{u})=-\left[\overline{\xi \mapsto \frac{\left(\beta^{\prime}(\xi) \xi-\beta(\xi)\right)}{\tilde{\mu}(\xi)}}\right] \bar{z}_{f}-\left[\overline{\xi \mapsto \frac{\left(\beta^{\prime}(\xi) \xi-\beta(\xi)\right) \tilde{c}(\xi)}{\tilde{\mu}(\xi)}}\right] \bar{\theta}
$$

In other words, the Young measures $\bar{\nu}=t \mapsto \bar{\nu}(t)$ satisfy the evolution equation:

$$
\partial_{t} \bar{\nu}+\partial_{x}(\bar{\nu} \bar{u}(t, x))=\partial_{\xi}\left(\frac{\xi}{\tilde{\mu}(\xi)}\left(\bar{z}_{f}(t, x)+\tilde{c}(\xi) \bar{\theta}(t, x)\right) \bar{\nu}\right)+\frac{\left(\bar{z}_{f}(t, x)+\tilde{c}(\xi) \bar{\theta}(t, x)\right)}{\tilde{\mu}(\xi)} \bar{\nu}
$$

in $\mathcal{D}^{\prime}((0, T) \times \mathbb{T} \times \mathbb{R})$

As for the constitutive laws, we note that, for fixed $n$ we may rewrite the definition of $z_{f}^{n}$ and $z_{h}^{n}$ as

$$
\frac{z_{f}^{n}+\tilde{c}\left(\rho^{n}\right) \theta_{n}}{\tilde{\mu}\left(\rho^{n}\right)}=\partial_{x} u_{n}, \quad \frac{z_{h}^{n}}{\tilde{\kappa}\left(\rho^{n}\right)}=\partial_{x} \theta_{n}
$$

Applying the compensated compactness argument of Lemma 6 - to pass to the limit in the left-hand sides of these equations - together with the weak convergences of $u^{n}, \theta^{n}$ we obtain that:

$$
\bar{z}^{f}(t, x)=\frac{1}{\left\langle\bar{\nu}(t)_{x}, \frac{1}{\tilde{\mu}}\right\rangle}\left(\partial_{x} \bar{u}(t, x)-\left\langle\bar{\nu}(t)_{x}, \frac{\tilde{c}}{\tilde{\mu}}\right\rangle \bar{\theta}(t, x)\right), \quad \bar{z}^{h}(t, x)=\frac{1}{\left\langle\bar{\nu}(t)_{x}, \frac{1}{\tilde{\kappa}}\right\rangle} \partial_{x} \bar{\theta}(t, x) .
$$

At the end of the day, this analysis yields the homogenized system (44)-(45)-(47) with constitutive laws (48).

The multiphase system is then obtained by plugging $x \mapsto \bar{\nu}(t)_{x} \in \mathcal{C}_{2}$ in these equations. To write it extensively, we go back to the modeling standpoint we presented as a motivation : the mixture of two phases + 
and - with constitutive equations denoted with corresponding indices. In that case, the generalized function $t \mapsto \bar{\nu}(t)_{x}$ is the combination $\alpha_{+}(t, x) \delta_{\rho_{+}(t, x)}+\alpha_{-}(t, x) \delta_{\rho_{-}(t, x)}$ and $\tilde{q}, \tilde{\kappa} \ldots$ are given by the convex combination of the values in both phases with weights $\alpha_{+}, \alpha_{-}$. For instance, we have:

$$
\left\langle\bar{\nu}(t)_{x}, \frac{1}{\tilde{\mu}}\right\rangle=\frac{\alpha_{+}(t, x)}{\mu_{+}}+\frac{\alpha_{-}(t, x)}{\mu_{-}} \quad\left\langle\bar{\nu}(t)_{x}, \frac{1}{\tilde{\kappa}}\right\rangle=\frac{\alpha_{+}(t, x)}{\kappa_{+}}+\frac{\alpha_{-}(t, x)}{\kappa_{-}} \ldots
$$

Furthermore, we can specify an equation for $\alpha_{+}$by choosing a test-function such that $\beta=1$ on the interval ranged by $\rho_{+}$and $\beta=0$ on the interval ranged by $\rho_{-}$. Similarly, we obtain an equation on $\alpha_{+} \rho_{+}$by choosing a test-function $\beta$ such that $\beta(\xi)=\xi$ on the interval ranged by $\rho_{+}$and $\beta=0$ on the interval ranged by $\rho_{-}$. This yields the system:

$$
\begin{aligned}
\partial_{t} \alpha_{+}+\alpha_{+} \partial_{x} u & =\frac{\alpha_{+} \alpha_{-}}{\alpha_{+} \mu_{-}+\alpha_{-} \mu_{+}}\left(\left[c_{P,+} \rho_{+}-c_{P,-} \rho_{-}\right] \theta+\left[\mu_{-}-\mu_{+}\right] \partial_{x} u\right], \\
\partial_{t}\left(\alpha_{+} \rho_{+}\right)+\partial_{x}\left(\alpha_{+} \rho_{+} u\right) & =0 \\
\partial_{t} \rho+\partial_{x}(\rho u) & =0 \\
\partial_{t}(\rho u)+\partial_{x}\left(\rho u^{2}\right) & =\partial_{x} z_{f} \\
\partial_{t}(q \theta)+\partial_{x}(q u \theta) & =\partial_{x} z_{h}+z_{f} \partial_{x} u
\end{aligned}
$$

with the constitutive equations:

$$
\begin{aligned}
\rho & =\alpha_{+} \rho_{+}+\alpha_{-} \rho_{-}, \\
q & =\alpha_{+} c_{V,+} \rho_{+}+\alpha_{-} c_{V,-} \rho_{-}, \\
z_{f} & =\frac{\mu_{-} \mu_{-}}{\alpha_{+} \mu_{-}+\alpha_{-} \mu_{+}} \partial_{x} u+\frac{\theta}{\alpha_{+} \mu_{-}+\alpha_{-} \mu_{+}}\left(\alpha_{+} c_{P,+} \mu_{-} \rho_{+}+\alpha_{-} c_{P,-} \mu_{+} \rho_{-}\right), \\
z_{h} & =\frac{\kappa_{+} \kappa_{-}}{\alpha_{+} \kappa_{-}+\alpha_{-} \kappa_{+}} \partial_{x} \theta .
\end{aligned}
$$

We remark that in this temperature-dependent case, we recover the intuitive extension of the isentropic case. In particular, in the volume fraction equation, we obtain the corresponding system changing the pressure term with the new pressure law.

Complementary remarks on a full analytical justification of the temperature-dependent multiphase system. In this last chapter, we provide a multiphase model with one velocity for viscous compressible fluids with temperature. We only focused on the necessary ingredients which enable to derive this system. It is important to remark that, in this analysis, the key-point is to derive a family of estimates for the fluid model under consideration (here the compressible Navier Stokes system with temperature) that allows discontinuous densities and smooth velocities. This analysis furnishes in the same time the family of solutions on which we can run the homogenization process, and highlights which terms will oscillate and which one will not in the computation of the homogenized system.

At the very end, we obtain the multiphase system by plugging that $x \mapsto \bar{\nu}(t)_{x} \in \mathcal{C}_{2}$ in the homogenized system. However, it is not clear that such an ansatz is preserved even in short time when solving (44)-(45)-(47)-(48). In the case of isentropic systems (see [2]), we propose to tackle this issue by a weak-strong uniqueness argument. We note that in the limiting process the fluid velocity-field enjoys the further property $\partial_{x} u \in L^{1}\left(0, T ; L^{\infty}(\mathbb{T})\right)$. This enables to construct solutions to (47) in the form of convex combinations of Dirac measures - with smooth weights $\left(\alpha_{i}\right)$ and centers $\left(\rho_{i}\right)$ - and then to show that any solution to $(47)$ is necessarily equal to this solution on a short time-interval.

\section{REFERENCES}

[1] D. Bresch, M. Hillairet. Note on the derivation of multicomponent flow systems. Proc. AMS, 143, 3429-3443, (2015). 
[2] D. Bresch, M. Hillairet. A compressible multifluid system with new physical relaxation terms. Ann. Sci. Éc. Norm. Supér. 52(4):255-295, no. 2, 2019.

[3] R. J. DiPerna. Measure-valued solutions to conservation laws. Arch. Rational Mech. Anal., 88(3):223-270, 1985.

[4] R. J. DiPerna, A. J. Majda. Oscillations and concentrations in weak solutions of the incompressible fluid equations. Comm. Math. Phys., 108(4):667-689, 1987.

[5] R. J. DiPerna, P.-L. Lions. Ordinary differential equations, transport theory and Sobolev spaces. Invent. Math., 98(3):511-547, 1989.

[6] D. A. Drew and S. L. Passman. Theory of multicomponent fluids, volume 135 of Applied Mathematical Sciences. SpringerVerlag, New York, 1999.

[7] E. Feireisl, A. Novotny, H. Petzeltova. On the existence of globally defined weak solutions to the Navier-Stokes equations. $J$. Math. Fluid Mech., 3, 358-392, (2001).

[8] D. Hoff. Global solutions of the Navier-Stokes equations for multidimensional compressible flow with discontinuous initial data. J. Differential Equations, 120(1):215-254, 1995.

[9] M. Ishii, T. Hibiki. Thermo-fluid dynamics of two-phase flow. Springer (2006).

[10] B. Kawohl. Global existence of large solutions to initial-boundary value problems for a viscous, heat-conducting, onedimensional real gas. J. Differential Equations, 58(1):76-103, 1985.

[11] A. V. Kazhikhov, V. V. Shelukhin. Unique global solution with respect to time of initial-boundary value problems for onedimensional equations of a viscous gas. Prikl. Mat. Meh., 41(2):282-291, 1977.

[12] P.-L. Lions. Mathematical topics in fluid mechanics, Vol. II: compressible models. Oxford Lect. Ser. Math. Appl. (1998).

[13] D. Serre. Variations de grande amplitude pour la densité dÕun fluide visqueux compressible. Phys. D, 48(1):113-128, (1991).

[14] L. Tartar. The compensated compactness method applied to systems of conservation laws. In Systems of nonlinear partial differential equations (Oxford, 1982), volume 111 of NATO Adv. Sci. Inst. Ser. C Math. Phys. Sci., pages 263-285. Reidel, Dordrecht, 1983.

[15] M. Youngs, D. Hoff. Existence of locally momentum conserving solutions to a model for heat-conducting flow. Z. Angew. Math. Phys., 64(2):283-310, 2013. 